\title{
CD248 as a bridge between angiogenesis and immunosuppression: a promising prognostic and therapeutic target for renal cell carcinoma
}

\author{
Shaojie Liu ${ }^{1 \# \wedge}$, Chao Xu" ${ }^{1 \#}$, Keying Zhang ${ }^{1 \#}$, Donghui Han ${ }^{1}, \mathrm{Fa} \mathrm{Yang}^{1}$, Yu Li $^{1}$, Xiaolong Zhao ${ }^{1}$, \\ Shanjin $\mathrm{Ma}^{2}$, Hongji $\mathrm{Li}^{1}$, Shiqi Lu ${ }^{3}$, Tong $\mathrm{Lu}^{1}$, Jiayu Zhang ${ }^{1}$, Weijun Qin ${ }^{1}$, Weihong Wen ${ }^{3}$, Bo Yang ${ }^{1}$ \\ ${ }^{1}$ Department of Urology, Xijing Hospital, Fourth Military Medical University, Xi'an, China; ${ }^{2}$ Department of Urology, Tangdu Hospital, Fourth \\ Military Medical University, Xi'an, China; 'nstitute of Medical Research, Northwestern Polytechnical University, Xi'an, China \\ Contributions: (I) Conception and design: S Liu, C Xu, K Zhang; (II) Administrative support: D Han, F Yang, B Yang; (III) Provision of study \\ materials or patients: Y Li, T Lu, W Qin, H Li; (IV) Collection and assembly of data: X Zhao, S Ma, W Wen; (V) Data analysis and interpretation: S \\ Lu, J Zhang; (VI) Manuscript writing: All authors; (VII) Final approval of manuscript: All authors. \\ \#These authors contributed equally to this work. \\ Correspondence to: Bo Yang. Department of Urology, Xijing Hospital, Fourth Military Medical University, Xi'an 710032 , China. \\ Email: xiybfmmu@163.com; Weihong Wen. Institute of Medical Research, Northwestern Polytechnical University, Xi'an 710072, China. \\ Email: weihongwen@nwpu.edu.cn; Weijun Qin. Department of Urology, Xijing Hospital, Fourth Military Medical University, Xi'an 710032, China. \\ Email: qinwj@fmmu.edu.cn.
}

Background: Renal cell carcinoma (RCC) is characterized by significant vascularization and immunogenicity, which contributes to drug resistance and immune escape. CD248, a pericytes marker in tumor vasculature, might help explain tumor microenvironment (TME) remodeling and serve as a novel therapeutic target.

Methods: Transcriptome data and clinical information of RCC patients were obtained from The Cancer Genome Atlas (TCGA) database. ESTIMATE and microenvironment cell population (MCP)-counter algorithms were adopted to calculate immune and stromal contents. The prognostic value of TME was evaluated via Kaplan-Meier and Wilcoxon signed rank test. Pearson's correlation coefficient was employed to explore the correlation between angiogenesis and TME, and the relationship between CD248 and TME or RCC progression. CD248 overexpression and vascular colocalization in RCC were confirmed via histology staining. The weighted gene coexpression network analysis (WGCNA) and enrichment analysis were performed to explore CD248-mediated regulatory mechanism in angiogenesis and TME remodeling. CD248-based drug response was predicted through CellMiner database.

Results: Tumor angiogenesis contributed to deteriorated RCC progression, which might be involved with immunosuppression. More specifically, upregulated immune checkpoints exhausted infiltrated $\mathrm{T}$ cells. CD248 overexpressed in RCC vessels correlated with TME and predicted a bad survival outcome. CD248 and coexpressed genes participated in angiogenesis and TME remodeling. Several clinical approved drugs that might inhibit CD248-mediated tumor promoting effects were selected.

Conclusions: CD248 appears to contribute to angiogenesis and immunosuppressive TME, and may thus be a promising prognostic and therapeutic target for RCC. CD248-based medication guidance might benefit RCC patients.

Keywords: Renal cell carcinoma (RCC); angiogenesis; tumor microenvironment (TME); CD248; immunosuppression

\footnotetext{
$\wedge$ ORCID: 0000-0002-5706-3841.
} 
Submitted Nov 15, 2021. Accepted for publication Dec 06, 2021.

doi: $10.21037 /$ atm-21-6271

View this article at: https://dx.doi.org/10.21037/atm-21-6271

\section{Introduction}

Renal cell carcinoma (RCC) is characterized by significant vascularization and immunogenicity, resulting in the bad clinical outcome of patients with RCC (1). Therefore, antiangiogenic treatment has been the standard treatment of RCC in the last decade, including vascular endothelial growth factor receptor 2 (VEGFR2) inhibitors like pazopanib and apatinib; multi-receptor tyrosine kinase inhibitors (TKIs) like sunitinib; and the mTOR inhibitor, everolimus $(1,2)$. However, regardless of whether antiangiogenic treatment alone or that combined with conventional treatment is applied, acquired resistance restricts the therapeutic efficacy $(3,4)$. The close relationship between angiogenesis and immunosuppressive tumor microenvironment (TME) was explicit that misshapen tumor vessels or increased pericyte coverage accelerate the invasion of tumor cells into basement membrane but prevent cytotoxic drug permeation, which are essential for acquired resistance $(5,6)$. More importantly, angiogenesis and proangiogenic factor contribute to immune cell recruitment and nourishment, which may increase metabolic complexity and is associated with therapy resistance (7). As a result, the therapies combining TKIs with immune-checkpoint inhibitors (ICIs) have shown increased therapeutic efficiency in advanced RCC (8). However, the underlying mechanisms tumor angiogenesis contributes to TME in RCC remain mysterious that an in-depth understanding is essential to exploring novel treatment strategies.

CD248, also named endosialin/tumor endothelial marker 1 (TEM1), was confirmed to be the marker of pericytes in neovascularization and tumor vasculature (9). Bagley et al. demonstrated that CD248 was specifically expressed in blood vessels during embryogenesis and tumorigenesis but not in normal mature vessels (10). Further studies have identified that CD248, as a helper receptor, responds to platelet-derived growth factor-BB (PDGF-BB) through phosphorylating platelet-derived growth factor receptor (PDGFR) and mitogen-activated protein kinase (MAPK) extracellular signal-regulated kinase $1 / 2$ (ERK1/2) to regulate the proliferation of pericytes (11). Following sprouting angiogenesis, CD248 promotes selective vessel regression and stabilization of remaining vessels through inducing the apoptosis and detachment of endothelial cells (EC) (12). The overexpression of CD248 has also been reported to be correlated with unfavorable prognoses in a variety of human malignancies. In TME, CD248 overexpressed in vessel-associated pericytes and myofibroblasts was shown to promote tumor growth and metastasis $(13,14)$. It has been shown that CD248 can interact with CD68 to recruit macrophages and regulate GAS6 expression in cancer-associated fibroblasts (CAFs) to facilitate macrophage M2 polarization and promote tumor growth (15). Whereas the exact biological function of CD248 remains unexplained in RCC to date.

Our previous study indicated that the overexpression of CD248 could reflect an immunosuppressive TME and is inversely correlated with the prognosis of patients with RCC (16). In this paper, we further analyzed the gene expression profiles and clinical information of RCC patients both from The Cancer Genome Atlas (TCGA) and clinical records. First, we defined tumor vessels as EC infiltration in RCC via the microenvironment cell population (MCP)counter algorithm and confirmed that EC infiltration was correlated with a suppressive TME. Thus, we highlighted significant vascularization as prominent component contributing to immunosuppressive TME. Inspired by the results of analyses for TCGA data and clinical pathological information we collected that were grouped by CD248 expression, we found that CD248 was significantly expressed around CD $31^{+}$ECs and positively correlated with microvascular density (MVD). Therefore, we speculate that CD248 might bridge the gap between angiogenesis and an immunosuppressive TME, and therefore may be promising prognostic and therapeutic target for RCC. To explore the underlying mechanisms, vascular-CD248-related differentially expressed genes (DEGs) were identified through an interaction method, based on which weighted gene coexpression network analysis (WGCNA) and enrichment analysis were performed to explore the CD248mediated regulatory mechanism in angiogenesis and suppressive TME remodeling. Finally, a CD248-based drug response was predicted through the CellMiner database, which may help guide future RCC therapy.

We present the following article in accordance with the REMARK reporting checklist (available at https://dx.doi. 
Table 1 Clinical pathological information of RCC patients

\begin{tabular}{|c|c|}
\hline Characteristics & Number (\%) \\
\hline \multicolumn{2}{|l|}{ Gender } \\
\hline Male & $42(52.50)$ \\
\hline Female & $38(47.50)$ \\
\hline \multicolumn{2}{|l|}{ Age, years } \\
\hline$<60$ & 39 (48.75) \\
\hline$\geq 60$ & $41(51.25)$ \\
\hline \multicolumn{2}{|c|}{ Histological grade } \\
\hline I & $30(37.50)$ \\
\hline II & 29 (36.25) \\
\hline III & $21(26.25)$ \\
\hline \multicolumn{2}{|l|}{ Tumor stage } \\
\hline $\mathrm{T} 1$ & $42(52.50)$ \\
\hline $\mathrm{T} 2$ & $20(25.00)$ \\
\hline T3 & $16(20.00)$ \\
\hline $\mathrm{T} 4$ & $2(2.50)$ \\
\hline \multicolumn{2}{|l|}{ Node stage } \\
\hline No & 69 (86.25) \\
\hline N1 & $11(13.75)$ \\
\hline \multicolumn{2}{|l|}{ Metastasis stage } \\
\hline MO & 78 (97.50) \\
\hline M1 & $2(2.50)$ \\
\hline \multicolumn{2}{|l|}{ TNM stage } \\
\hline I & 39 (48.75) \\
\hline II & $18(22.50)$ \\
\hline III & $19(23.75)$ \\
\hline IV & $4(5.00)$ \\
\hline
\end{tabular}

RCC, renal cell carcinoma; TNM, tumor-node-metastasis.

org/10.21037/atm-21-6271).

\section{Methods}

\section{Raw data and specimen}

First, 895 RCC and 128 normal samples were downloaded from TCGA database (http://portal.gdc.cancer.gov/). The transcriptome data profile and clinical information were integrated through ID numbers. "Limma" package in $\mathrm{R}$ software (The R Foundation for Statistical Computing, Vienna, Austria) was used to calculate the average gene expression from the transcriptome data profile. $\mathrm{R}$ software was also used to process and analyze the clinical information. The pathological information of 80 RCC patients was obtained from the Urology Department retrospectively with the corresponding paraffin-embedded RCC tissues and adjacent normal tissues sections from the Pathology Department of Xijing Hospital, Fourth Military Medical University (Xi'an, China). The 80 RCC patients who received surgery but not chemotherapy were included in this study. The pathological information of the 80 patients was shown in Table 1. All procedures performed in this study involving human participants were in accordance with the Declaration of Helsinki (as revised in 2013). The study was approved by the Ethics Committee of the Xijing Hospital, Fourth Military Medical University (No. KY20162088-1). Individual consent for this retrospective analysis was waived.

\section{Calculation of the abundance of RCC-infiltrating immune and stromal cells}

The MCP-counter algorithm (provided by TIMER 2.0; http://timer.cistrome.org/) was used to calculate the abundance of RCC-infiltrating immune and stromal cells. According to the transcriptome data profile, the abundance of 8 kinds of immune cell types, including $\mathrm{T}$ cells, $\mathrm{CD} 8^{+} \mathrm{T}$ cells, cytotoxic lymphocytes, B lineage lymphocytes, natural killer (NK) cells, monocytic lineage, myeloid dendritic cells (MDCs), neutrophils, and 2 kinds of stromal cell types (EC and fibroblasts) were quantified. The scale of fraction represented the infiltration degree of the cells in the TME. The transcriptome data were normalized.

\section{Survival analysis}

The correlation between survival and EC infiltration or CD248 was analyzed using "survival" and "survminer" R packages. The Kaplan-Meier method was applied in plotting the survival curves. The clinical endpoint was defined as the death of the patients. The difference of overall survival (OS) between the defined high and low groups of ECs infiltration or CD248 expression was analyzed by Wilcoxon rank sum 
test, with a $\mathrm{P}<0.05$ being considered significant.

\section{Calculation of ImmuneScore, StromalScore and ESTIMATEScore}

The ESTIMATE algorithm was used to calculate the ImmuneScore and StromalScore of RCC samples, which were positively correlated with the amount of immune and stromal components in TME. The ESTIMATEScore was the sum of ImmuneScore and StromalScore. The count matrix was normalized and $\log _{2}$ transformed.

\section{Histology staining}

Immunohistochemistry (IHC) staining and immunofluorescent (IF) staining were performed to examine and localize CD248 expression in RCC tissues and adjacent normal tissues. The primary antibodies used were as follows: CD248 (\#ab204914, Abcam, Cambridge, UK), CD31 (\#89C2, Cell Signaling Technology, USA), CD3 (\#2100567, eBioscience, USA), CD206/MRC1 (\#24595, Cell Signaling Technology, USA). The second antibodies were as follows: goat anti-rabbit immunoglobin [IgG; H\&L; horse radish peroxidase (HRP); \#ab6721, Abcam], donkey anti-rabbit IgG (\#ab150076, Abcam), donkey anti-mouse IgG (\#ab150105, Abcam). Nuclei were stained with DAPI (\#C1002, Beyotime, Shanghai, China). Quantification was performed according to the percentage and intensity in IHC staining and the percentage of the positive area in the IF staining using Image J v1.52a (NIH, Bethesda, MD, USA).

\section{DEGs analysis}

We divided samples into 2 groups according the levels of EC infiltration and CD248 expression. DEGs between the 2 groups were selected with the following threshold: corrected $\mathrm{P}<0.05$ and $\left|\log _{2} \mathrm{FC}\right|>1$. The "Pheatmap" package in $\mathrm{R}$ software was used to draw the heatmaps of the DEGs. The upregulated and downregulated DEGs in the EC infiltration gene set was intersected with those in the CD248 expression gene set for further analyses, the results of which were visualized by Venn 2.1.0 (http://bioinfogp. cnb.csic.es/tools/venny/index.html).

\section{WGCNA for vascular-CD248-related DEGs}

The intersection method-selected vascular-CD248-related
DEGs were grouped into modules via hierarchical average linkage clustering. The correlation between RCC clinical phenotype and different color-marked modules was drawn by the module trait heatmap. A $\mathrm{P}<0.05$ was considered significant. The prognostic modules were visualized with Cytoscape 3.6.0 and further analyzed with Gene Ontology (GO) and Kyoto Encyclopedia of Genes and Genomes (KEGG) enrichment analysis.

\section{GO and KEGG enrichment analysis and gene set enrichment analysis (GSEA)}

GO and KEGG enrichment analyses for the DEGs were conducted using the "clusterprofiler" package in R. A corrected $\mathrm{P}<0.05$ indicated that the GO terms and KEGG pathways were significantly enriched. GSEA software obtained from the Broad Institute was performed to conduct GSEA for the KEGG gene sets. A corrected $\mathrm{P}<0.05$ indicated statistical significance. The data of the gene sets were $\log _{2}$ transformed.

\section{CD248-based drug response prediction}

Clinically approved drugs that might inhibit CD248mediated tumor-promoting effects were primarily screened from CellMiner version 2.6 (http://discover.nci.nih.gov/ cellminer/home.do). Drugs with a $\mathrm{P}<0.05$ were considered to be significantly correlated with CD248. A correlation coefficient $<0$ and $>0$ was considered to indicate a negative and positive correlation, respectively; meanwhile, $0.3<$ | correlation coefficient $\mid \leq 0.5$ indicated a low response of the drugs to CD248.

\section{Statistical analysis}

Statistical analyses were performed using GraphPad Prism 8.0 (GraphPad Software, Inc., San Diego, CA, USA). Transcriptome data were analyzed by $t$-test and nonparametric test. Differential analyses between the defined high and low groups of EC infiltration and CD248 expression were conducted by Wilcoxon rank sum test. Pearson's correlation coefficient was applied in correlation analysis between TME scores and ECs infiltration or CD248 expression. Chi-square test was used to analyze the relationship between CD248 expression and pathological information of the 80 RCC patients. A $\mathrm{P}<0.05$ was considered statistically significant. 


\section{Results}

\section{Angiogenesis contributed to $R C C$ progression through immunosuppressive TME}

To explore the relationship between TME and RCC survival outcome, we performed Kaplan-Meier survival analysis for ImmuneScore, StromalScore and ESTIMATEScore. The results indicated that high TME scores associated with poor clinical outcomes ( $\mathrm{P}<0.001$, Figure $1 A)$, deteriorated tumornode-metastasis (TNM) grade, and pathological stage in patients with RCC $(\mathrm{P}<0.05$, Figure $1 B)$. Furthermore, high tumor vessel infiltration (i.e., angiogenesis) promoted RCC progression, and EC infiltration positively correlated with TME score and TNM grade $(\mathrm{P}<0.05$, Figure $1 C, 1 D$; $\mathrm{P}<0.05$, Figure $\mathrm{S} 1 \mathrm{~A})$. EC infiltration associated with the recruitment of $\mathrm{T}$ cells, $\mathrm{CD}^{+} \mathrm{T}$ cells, cytotoxic lymphocytes, NK cells, monocytic lineage, MDCs, and fibroblasts, but not B lineage or neutrophils. Interestingly, the correlation between StromalScore and TME was similar to that of ECs, indicating that vasculature might be the prominent component of RCC stroma $(\mathrm{P}<0.05$; Figure $1 E, 1 F)$. The relationships between TME and RCC prognosis (i.e., TNM grade, pathological stage, and histological grade) were visualized with a heatmap (Figure S1B). Additionally, several immune checkpoints were upregulated in the high EC-infiltration group, as well as the biomarkers of M2 macrophages (i.e., CD163, MSR1, and MS4A4A; $\mathrm{P}<0.05$; Figure $1 G$ ), indicating that EC-recruited $\mathrm{T}$ cells might be exhausted and that the TME was predominantly immunosuppressive.

\section{CD248 contributed to RCC progression through angiogenesis}

The survival outcome of RCC patients was decreased with the overexpression of CD248 ( $\mathrm{P}<0.001$; Figure $2 A)$. Similarly, upregulated CD248 was associated with tumor and node stage pathological stage, and TNM stage but not gender, age and metastasis $(\mathrm{P}<0.05$; Figure $2 B)$. CD 248 also displayed a positive correlation with TME, including ImmuneScore, StromalScore, and ESTIMATE Score $(\mathrm{P}<0.05$, Figure 2C,2D). GSEA of KEGG indicated that CD248 was involved with multiple angiogenesis pathways, including janus kinase/signal transducer and activator of transcription (JAK/STAT) signaling pathway, MAPK signaling pathway, Notch signaling pathway, transforming growth factor $\beta$ (TGF- $\beta$ ) signaling pathway and vascular smooth muscle contraction (Figure 2E). Clustering analysis revealed that proangiogenic genes (i.e., $V E G F A$, $T E K, S O X 18, C D H 5$, and SOX17) were activated in the CD248 high expression group (Figure $2 F$ ), indicating that CD248 might contribute to RCC progression through proangiogenic signals.

\section{Vascular-expressed CD248 contributed to the immune regulation of TME}

To confirm the relationship between CD248 and angiogenesis, IHC staining of the 80 RCC patients for CD248 expression was performed. As the representative images shown in Figure 3A,3B, CD248 was over-expressed in RCC but not adjacent normal tissues (normal: 2.58 0.26 ; RCC: $15.65 \pm 1.54 ; n=3$ ). Furthermore, IHC staining analysis confirmed the significant correlation between CD248 expression and pathological stage, tumor stage and TNM stage as well (Table 2). CD248 was also positively correlated with MVD (Figure 3C). Representative IF staining further verified that CD248 was expressed surrounding CD $31^{+}$ ECs (Figure 3D). A heatmap for vascular-expressed CD248 was constructed for visualization of the clinical information and TME. This clarified that vascular-expressed CD248 negatively correlated with $\mathrm{T}$ cell infiltration and positively correlated with the infiltration of $\mathrm{ECs}, \mathrm{CD}^{+} \mathrm{T}$ cells, cytotoxic lymphocytes, NK cells, monocytic lineage, MDCs, stromal fibroblasts, and TME scores (Figure S2), indicating that vascular-expressed CD248 contributed to the immune regulation of TME. According to the results shown in Figure $1 F, 1 G$, angiogenesis contributes to immunosuppressive TME mainly characterized by decreased $\mathrm{T}$ cell infiltration and M2 macrophage polarization. Therefore, we further perform IHC staining for RCC tissues targeting CD3 for quantitative analysis of T cell and CD206 for M2 macrophage. Correspondingly, the RCC tissues with overexpression of CD248 showed decreased $\mathrm{T}$ cell and increased M2 macrophage infiltration (Figure 3E,3F).

\section{Vascular-CD248-related DEGs contributed to RCC angiogenesis}

To explore the regulatory mechanism of vascularexpressed CD248 on RCC angiogenesis, 2,864 DEGs based on CD248 expression and 2,611 DEGs based on ECs infiltration were obtained, with the top 100 DEGs being shown in Figure $4 A, 4 B$, respectively. Then, the vascular-CD248-related DEGs were identified through 

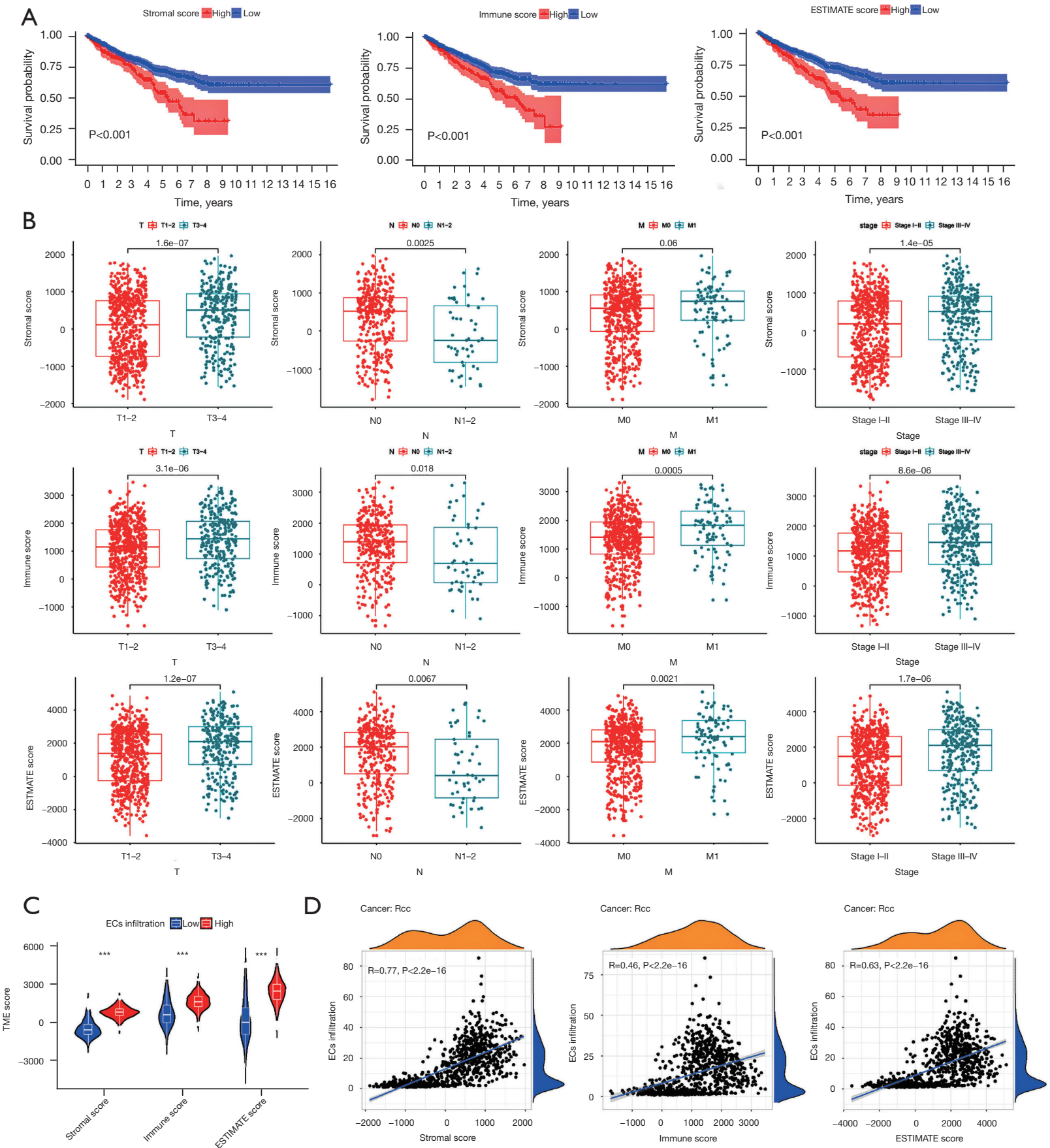


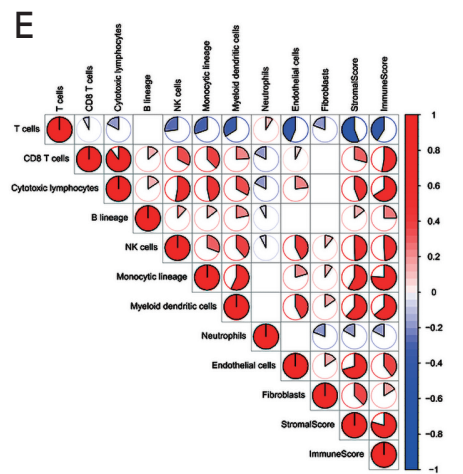

$\mathrm{F}$

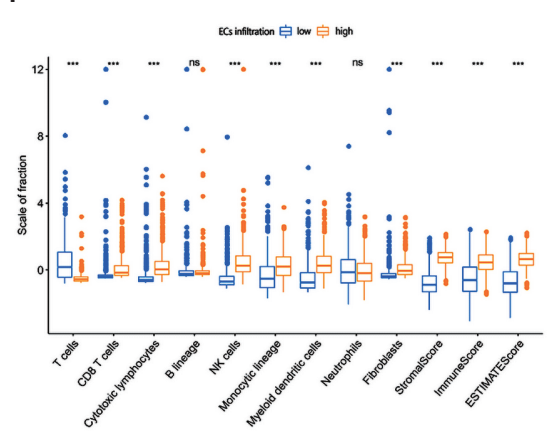

G

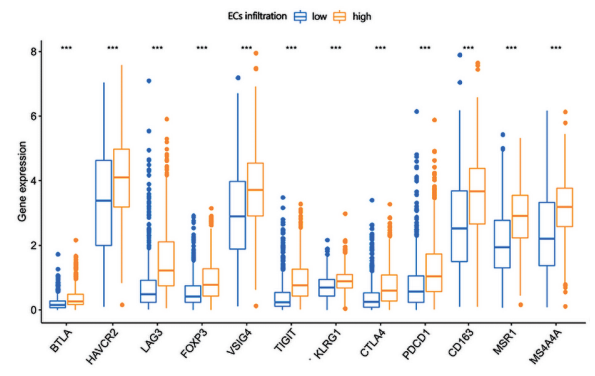

Figure 1 Tumor vasculature led to a poor prognosis and immunosuppressive TME in RCC. (A) Kaplan-Meier survival analysis for RCC patients of stromal score, immune score, and ESTIMATE score with high and low scores. (B) Distribution of TME scores in TNM classification and RCC stage. (C) Statistic analysis for RCC patients of high and low degree of EC infiltration in TME scores. (D) The correlation between EC infiltration and TME scores. (E) The correlation of immune and stromal cells with TME scores. (F) Statistical analysis for the high and low degree of EC infiltration in TME components and scores. (G) Gene expression of ICI molecules and M2 macrophage markers in RCC patients of a high and low degree of EC infiltration. *** $\mathrm{P}<0.0001$. RCC, renal cell carcinoma; TME, tumor microenvironment; EC, endothelial cells; ICI, immune-checkpoint inhibitor; ns, no significance.

interaction analysis, revealing 1,079 upregulated DEGs and 1,181 downregulated DEGs (Figure 4C). GO enrichment analysis indicated that vascular-CD248-related DEGs were associated with the GO terms of vasculature development, including vascular wound healing, vasculogenesis, positive regulation of smooth muscle contraction, kidney vasculature development, and others (Figure 4D). KEGG enrichment analysis revealed the top 20 pathways enriched by vascularCD248-related DEGs. Notably, several angiogenesisrelated pathways were significantly enriched, such as vascular smooth muscle contraction, PI3K-Akt signaling pathway, hypoxia-induced factor-1 (HIF-1) signaling pathway, Rap1 signaling pathway, and others (Figure $4 E$ ).

\section{Vascular-CD248-related DEGs contributed to angiogenesis and immune regulation}

To explore the regulatory mechanism of vascular-expressed CD248 on immune regulation, WGCNA of vascularCD248-related DEGs was performed. As a result, 13 coexpression gene modules were divided via hierarchical average linkage clustering (Figure $5 A$ and Figure S3). A module-trait heatmap indicated that modules marked with black, blue, green-yellow, and yellow were significantly associated with RCC progression $(\mathrm{P}<0.05$; Figure $5 B)$. The interactions among the 4 selected modules were visualized with a regulatory network, and the prominent GO function of each module was labeled (Figure 5C). The blue module annotated with angiogenesis were visualized (Figure 5D). Furthermore, GO and KEGG enrichment analysis was performed to explore the mechanisms of the modules related to RCC progression (Figure 5E, 5F). The yellow module annotated with immunity was also visualized and the corresponding GO and KEGG enrichment analysis was performed as well (Figure 5G-5I). A regulatory network of the black module annotated with stromal and green module annotated with metabolism and the corresponding GO and KEGG enrichment analysis were also conducted (Figure S4). As shown in Figure 57, the interactions among CD248, the top 20 hub genes most closely related to the others of the blue and the yellow module, were visualized. Moreover, 7 angiogenesis-related genes and 15 immunerelated genes were included in the regulatory network, in which CD248 was at start position, indicating that vascularexpressed CD248 contributed to immune regulation.

\section{CD248-based drug response prediction}

Having confirmed that CD248 plays a pivotal role in RCC progression via angiogenesis and immune regulation, we screened for several clinically approved drugs that might inhibit CD248-mediated tumor-promoting effects (Figure 6). Zoledronate, nelarabine, epirubicin, and temsirolimus positively responded to CD248. Conversely, CD248 expression might reduce the effectiveness of pralatrexate, trametinib, 7-ethyl-10-hydroxycamptothecin, 

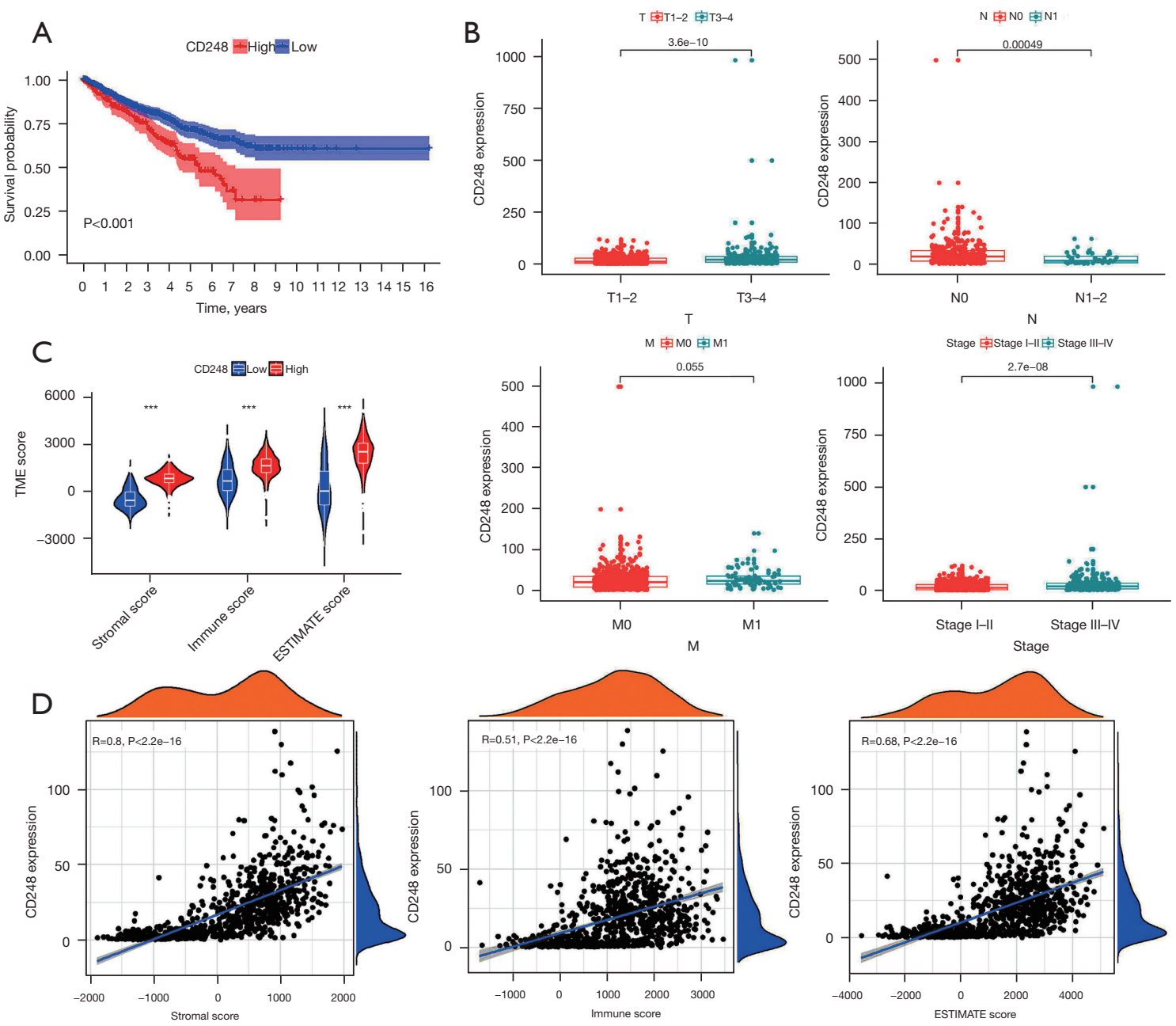


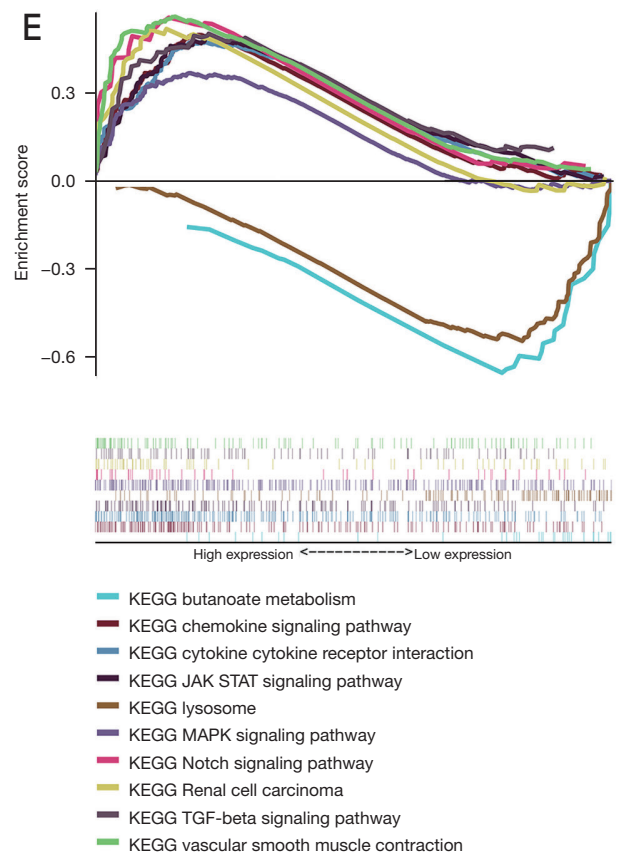

$\mathrm{F}$

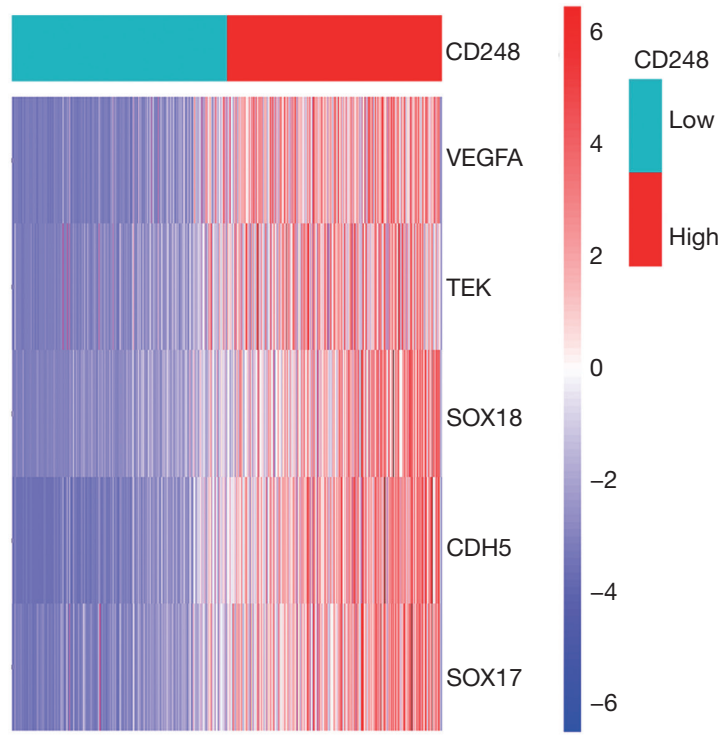

Figure 2 CD248 was associated with a poor prognosis and was involved in angiogenesis in RCC. (A) Kaplan-Meier survival analysis for RCC patients of CD248 with high and low expression. (B) Distribution of CD248 expression in TNM classification and stage of RCC. (C) Statistical analysis for RCC patients of high and low expression of CD248 in TME scores. (D) The correlations between CD248 expression and TME scores. (E) The hallmark enrichment of high and low expression of CD248 in RCC patients according to GSEA. (F) The heatmap of angiogenic factors in RCC patients with high and low CD248 expression. ${ }^{* *}, \mathrm{P}<0.0001$. RCC, renal cell carcinoma; TME, tumor microenvironment; TNM, tumor-node-metastasis; GSEA, gene set enrichment analysis.

cobimetinib and vismodegib.

\section{Discussion}

There are over 400,000 new cases and 175,000 deaths of RCC worldwide every year. The incidence of RCC is more common in males, being twice as that which occurs in females (17). The treatment of RCC drastically advanced in the past decade during which targeted therapies and ICIs have been applied in the front-line and second-line settings, demonstrated survival benefit in advanced RCC. Although RCC is susceptible to immunotherapy, immune tolerance and immune escape are inevitable due to the complex vascularization and immunogenicity. Therefore, an in-depth understanding of tumor angiogenesis and TME is essential to exploring novel treatment approached for RCC. For one, abnormally distributed tumor vessels with increased tortuosity and permeability can facilitate epithelial cell invasion into blood while inhibiting $\mathrm{T}$ cell infiltration (18). For another, proangiogenic factors leading to vascular abnormalities in tumor can directly and indirectly contribute to an immunosuppressive TME through anaerobic metabolism-induced low $\mathrm{PH}$ and immunosuppressive cell recruitment, including that of immature dendritic cells (DCs), regulatory T (Treg) cells, and M2-type tumorassociated macrophages (TAMs) $(6,19)$. Moreover, VEGF inhibition-mediated angiogenesis normalization can reverse the transition of antigen-presenting cells (APCs) toward a tolerant phenotype involved with T cell suppression (18). Indeed, the existence of close relationship between tumor angiogenesis and immunosuppressive TME remodeling is certain; however, the interaction mechanism remains largely unknown.

In the present study, we demonstrated that both immune and stromal components are positively correlated with poor prognosis. More precisely, the infiltration of ECs, $\mathrm{CD}^{+} \mathrm{T}$ cells, cytotoxic lymphocytes, NK cells, monocytic lineage, MDCs, and stromal fibroblasts were significantly increased in the stroma of RCC. An increased infiltration of ECs (a marker of vascularization) could promote tumor angiogenesis and enhance immune response via immune cell infiltration. However, increased $\mathrm{CD} 8^{+} \mathrm{T}$ cell infiltration 


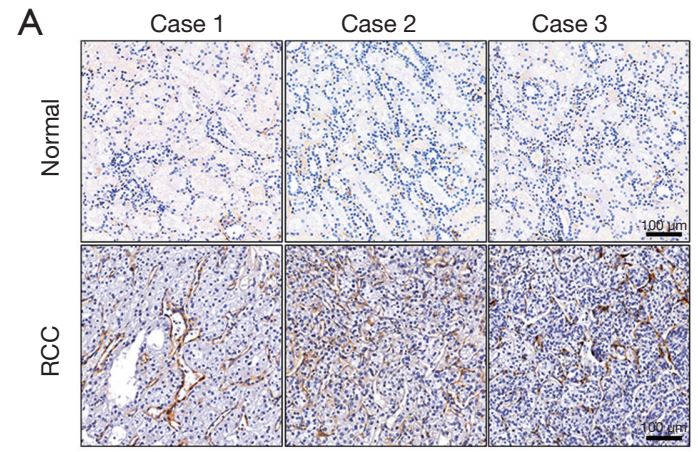

B
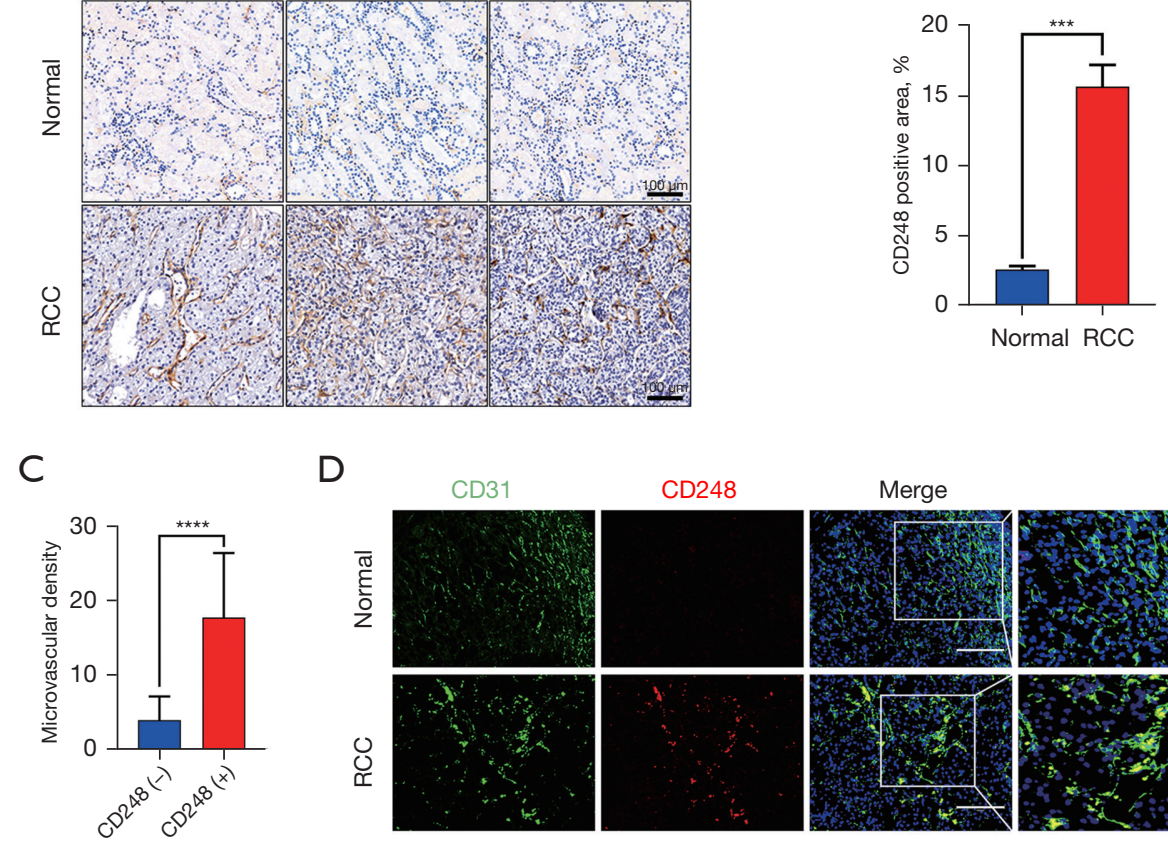

D

E
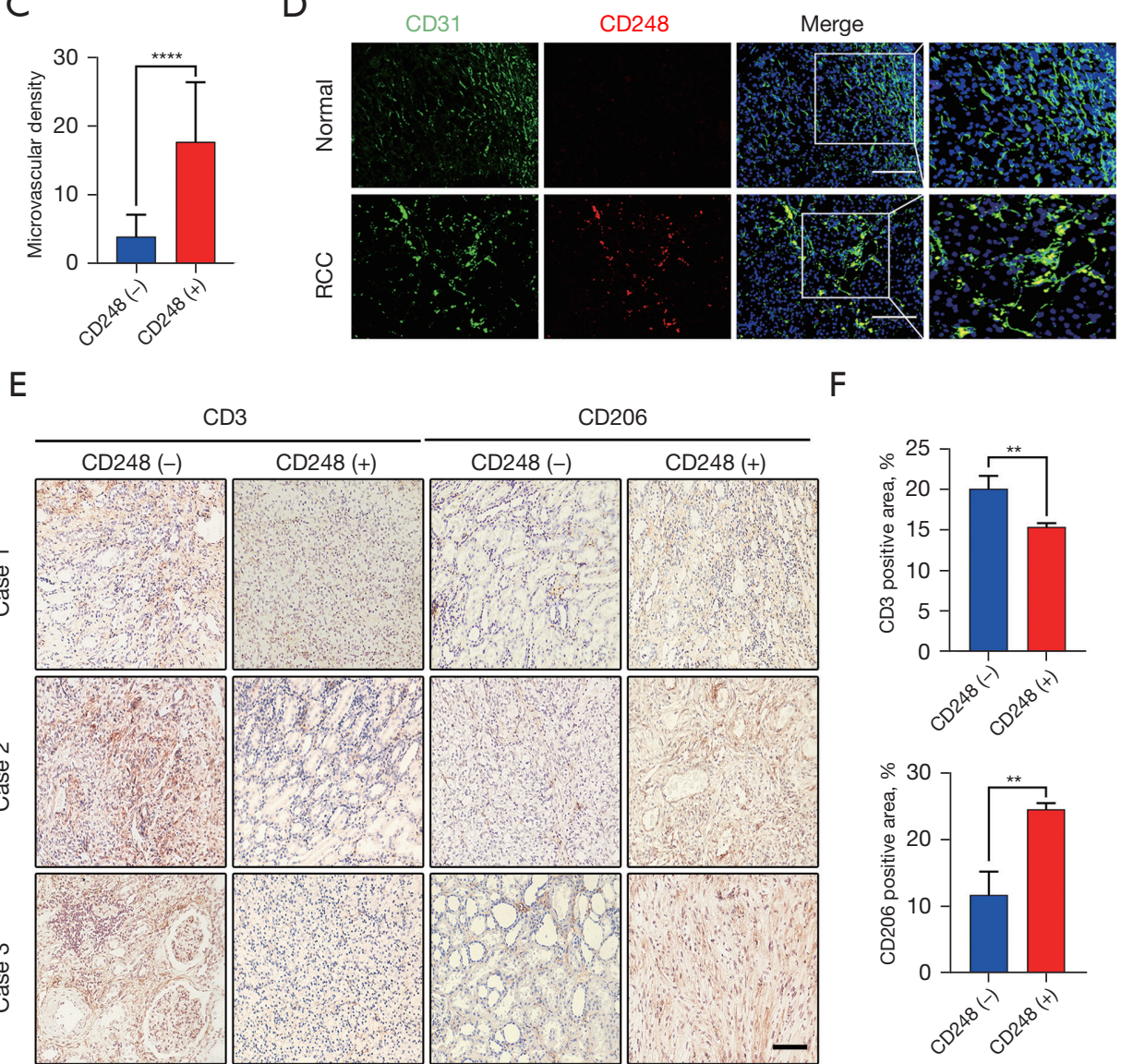

$\mathrm{F}$
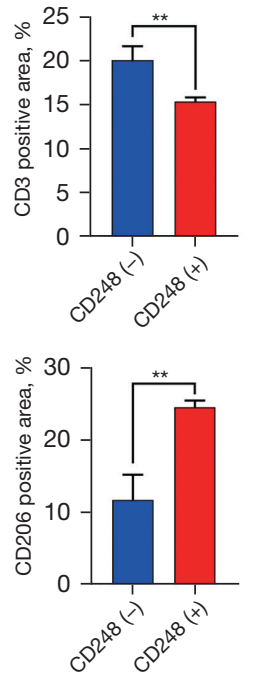

Figure 3 CD248 was expressed in RCC vasculature and involved in an immunosuppressive TME. (A) IHC staining of CD248 in RCC and adjacent normal tissues (scale bar, $100 \mu \mathrm{m}$ ). (B) Quantitative analysis for the data derived from the IHC staining. (C) MVD of RCC tissues with positive and negative expression of CD248. (D) Representative IF costaining of CD248 and CD31 in RCC (scale bar, $100 \mu \mathrm{m}$ ). (E) IHC staining of CD3 and CD206 in RCC tissues with positive and negative expression of CD248 (scale bar, $100 \mu \mathrm{m}$ ). (F) Quantitative analysis for the data from the IHC staining in (E). **, $\mathrm{P}<0.01$; ${ }^{* * *}, \mathrm{P}<0.001$; ${ }^{* * *}, \mathrm{P}<0.0001$. RCC, renal cell carcinoma; TME, tumor microenvironment; IHC, immunohistochemistry; MVD, microvascular density; IF, immunofluorescent. 
Table 2 The relationship between CD248 and pathological information of RCC patients

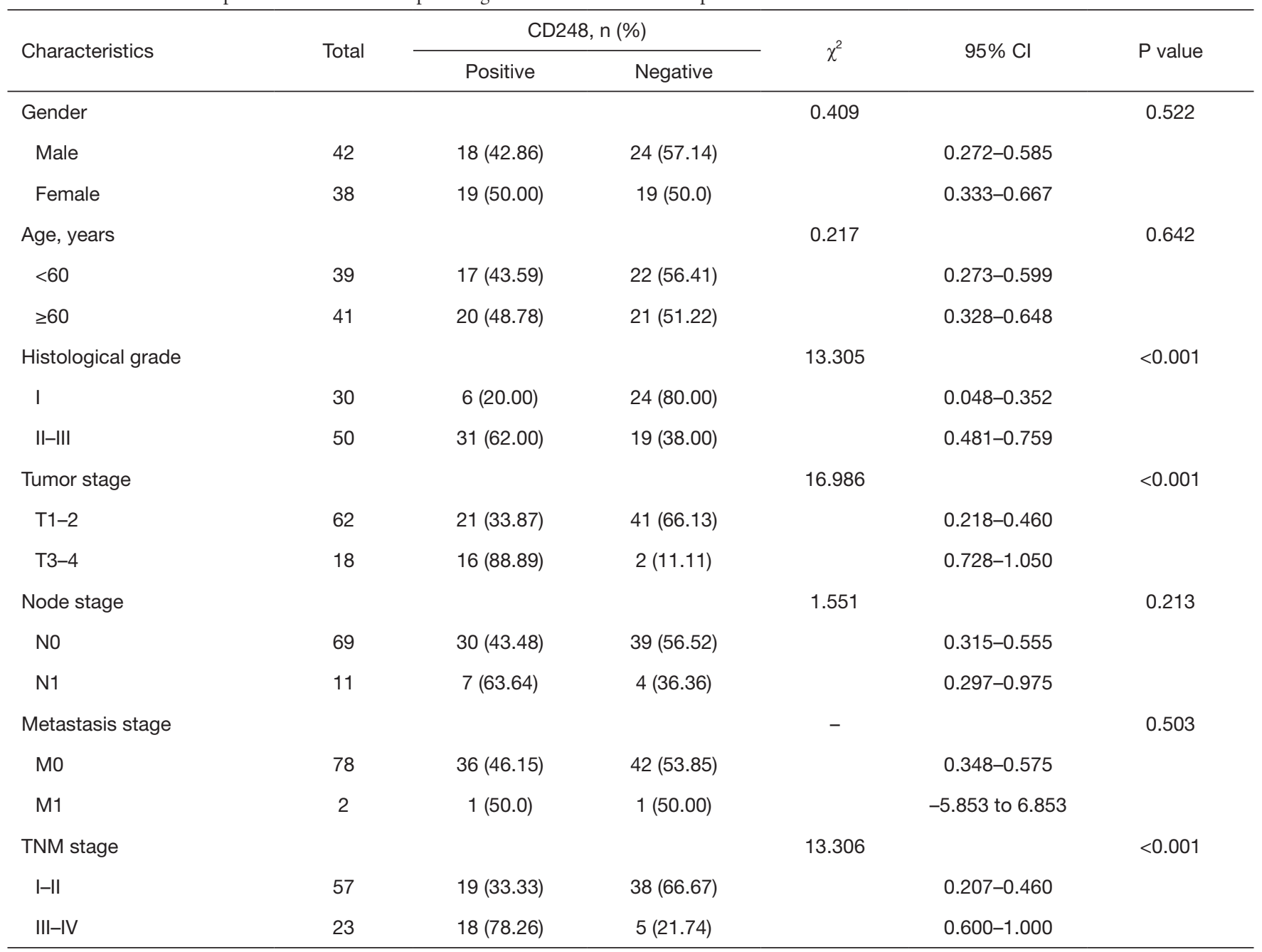

$\mathrm{Cl}$, confidence interval; CD, cluster differentiation; TNM, tumor-node-metastasis; RCC, renal cell carcinoma.

was associated with a worse RCC survival outcome rather than an antitumor effect, which was consistent with a previous study (20). We speculated that infiltrated $\mathrm{T}$ cells might be exhausted in an immunosuppressive TME. First, prominent immune-suppressive factors (i.e., VEGF, iterleukin-10, and TGF- $\beta$ ) in RCC TME may transform infiltrated MDCs to the tolerant phenotype and promote immunosuppression (21). In addition, infiltrated monocytes may have the markers of M2 macrophages, which can not only directly stimulate angiogenesis and tumor cells proliferation, but also produce immunosuppressive cytokines and extracellular matrix remodeling enzymes to promote immunosuppressive TME (22). Overexpressed immune checkpoints also contribute to $\mathrm{T}$ cell exhaustion. Therefore, $\mathrm{T}$ cell exhaustion and increased infiltration of immunosuppressive cells ascribed to RCC vascularization might lead to an immunosuppressive TME and tumor progression.

CD248 expressed in tumor stromal fibroblasts and vessels could promote tumor cell migration and invasion $(23,24)$. Indeed, pericytes expressing CD248 have been shown to facilitate tumor cell intravascular migration in a cell contact-dependent manner (14). Further study reported that proangiogenic factors (i.e., TGF- $\beta$, PDGF$\mathrm{BB}$, and Notch pathways) were altered in mice lacking the CD248 cytoplasmic domain, which led to tumor growth reduction (25). Notably, CD248 not only contributes to tumor progression through angiogenesis, but also contributes to tumor-promoting immune regulation. Immunotherapy targeting CD248 has proven to be effective 
A

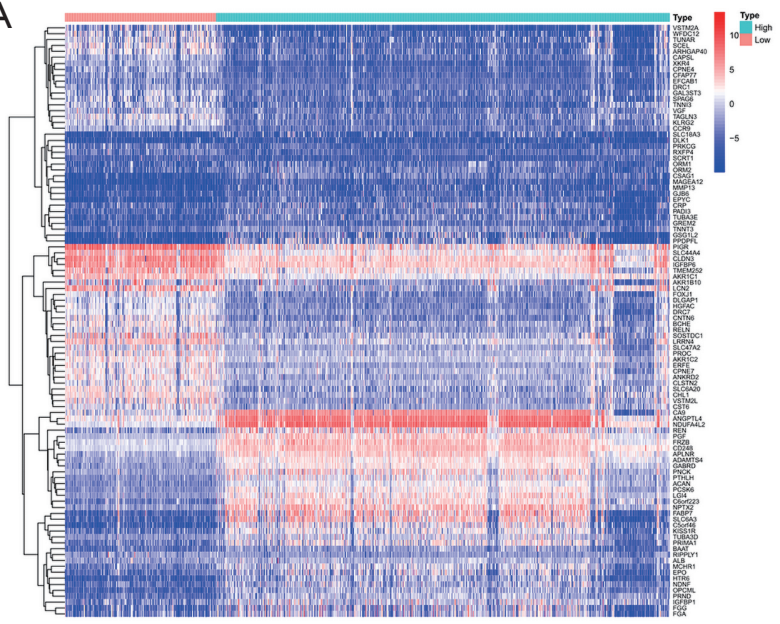

C

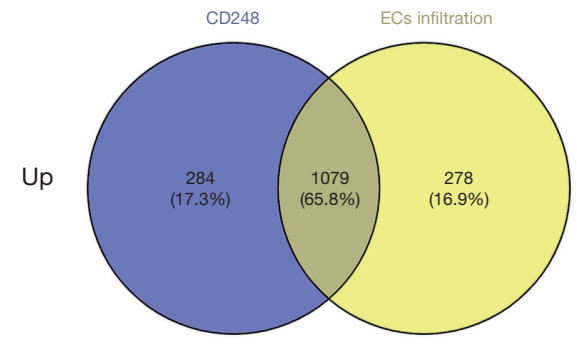

$\mathrm{D}$

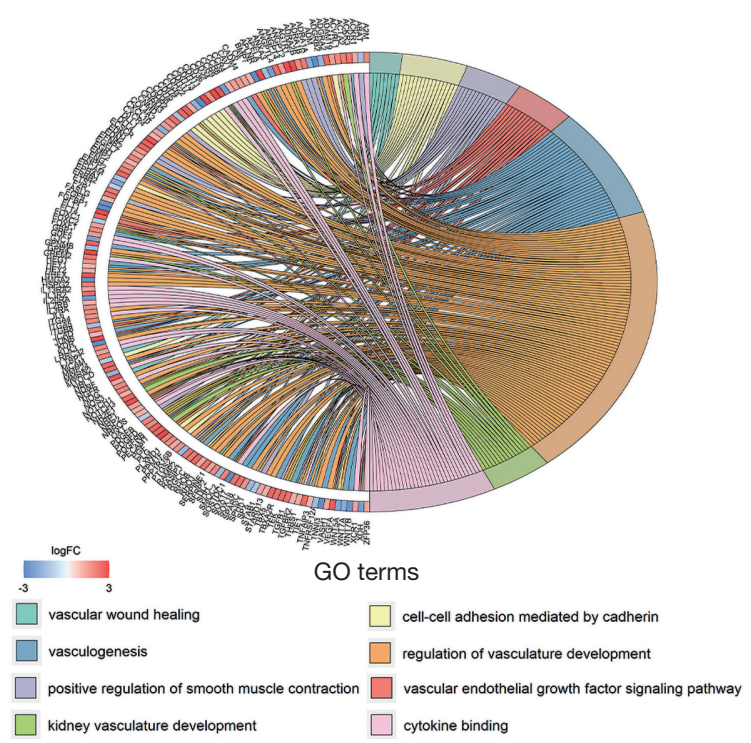

B
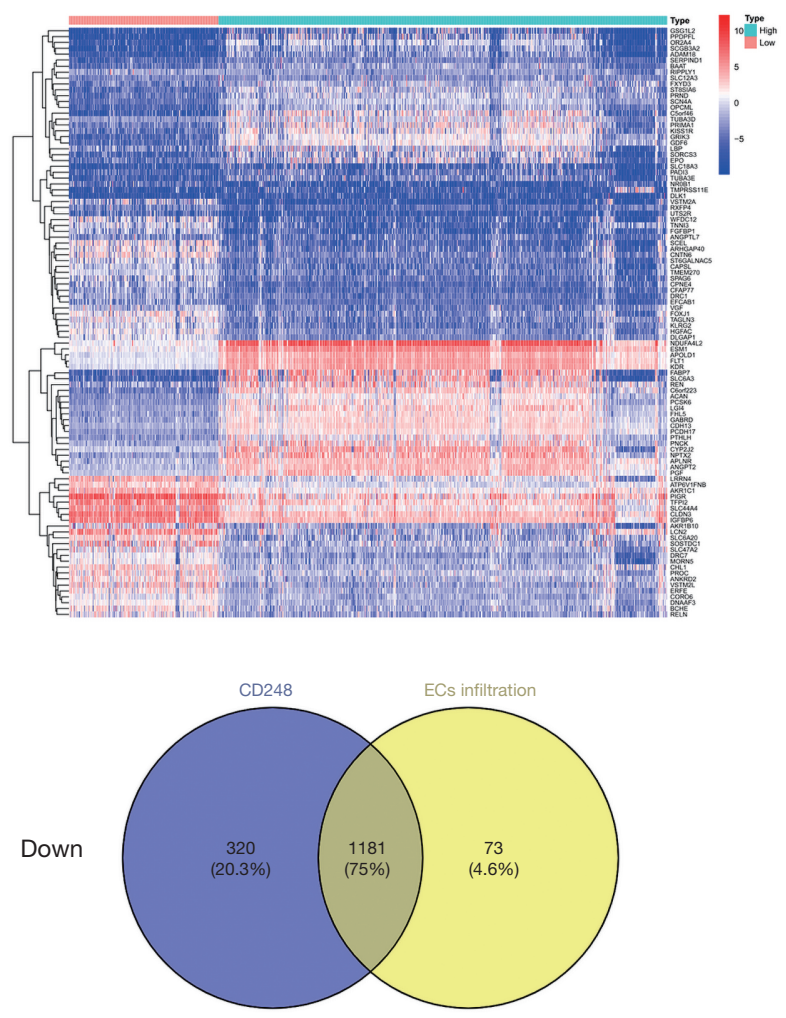

$E$

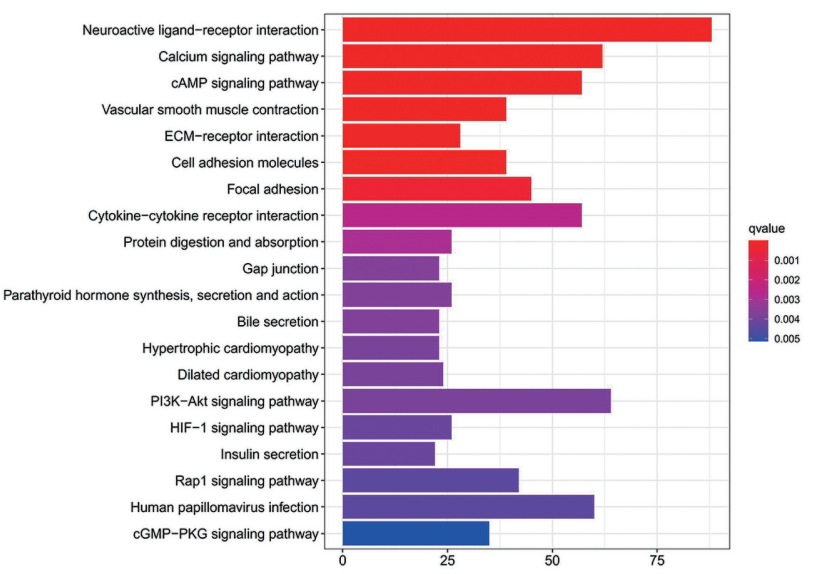

Figure 4 Acquisition and enrichment analyses of GO and KEGG for DEGs. (A,B) Heatmap for DEGs generated by a comparison of the high versus low group in EC infiltration and CD248 expression. (C) Venn plots for the shared upregulated and downregulated DEGs by EC infiltration and CD248 expression. (D,E) GO (D) and KEGG (E) enrichment analysis for 2,260 DEGs; terms with P and q $<0.05$ were considered significant. GO, Gene Ontology; KEGG, Kyoto Encyclopedia of Genes and Genomes; DEGs, differentially expressed genes; EC, endothelial cells. 

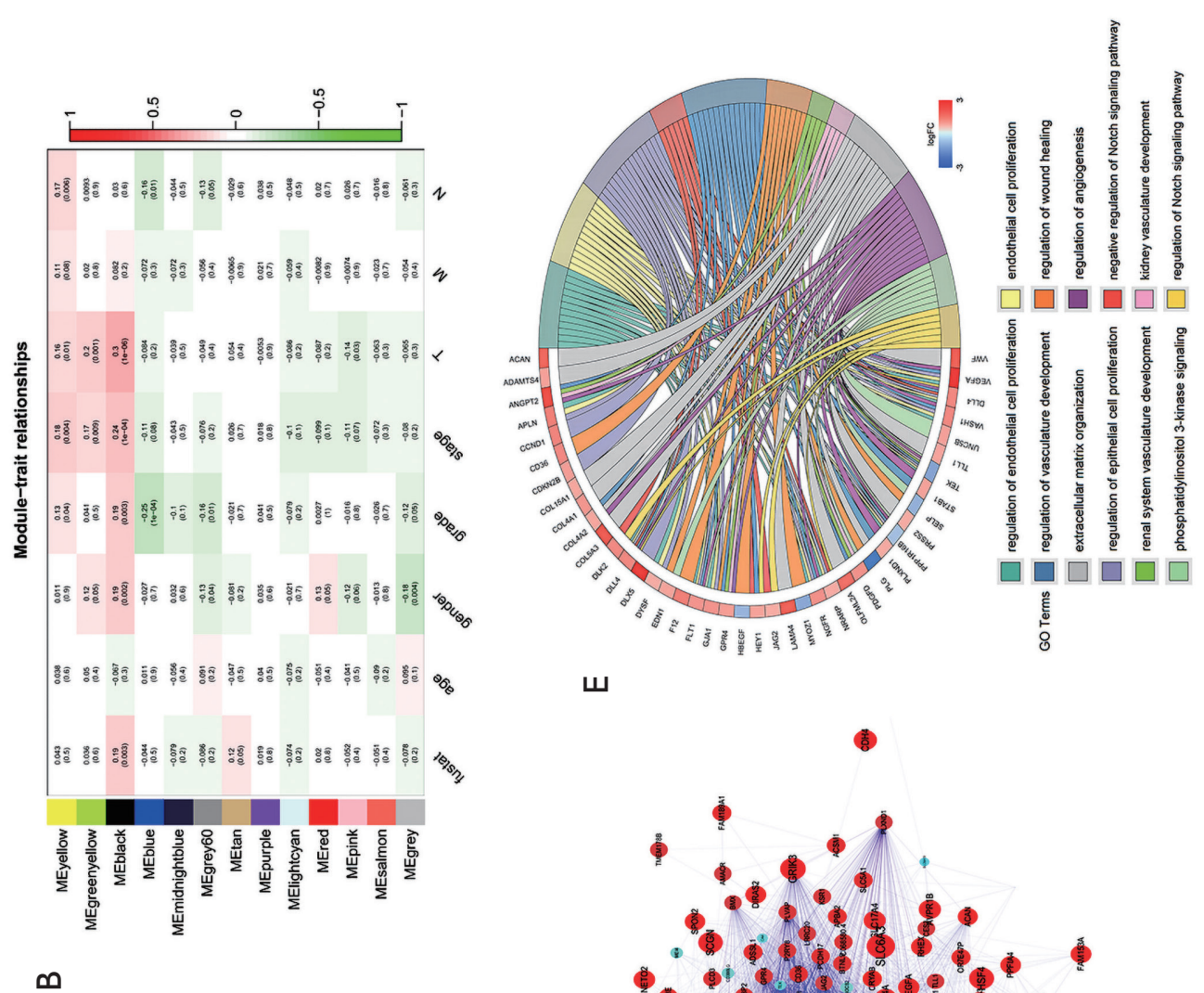

ш
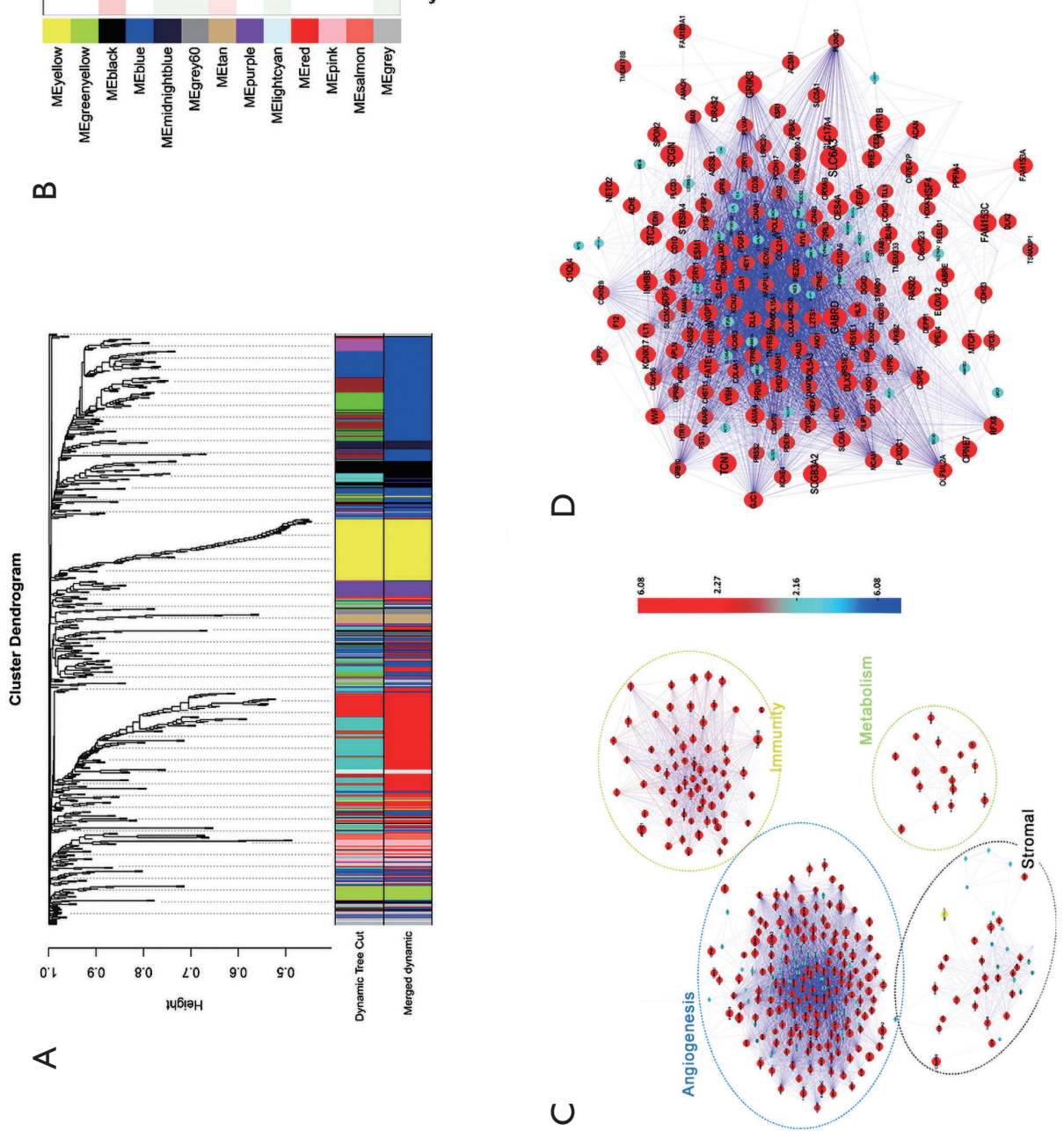

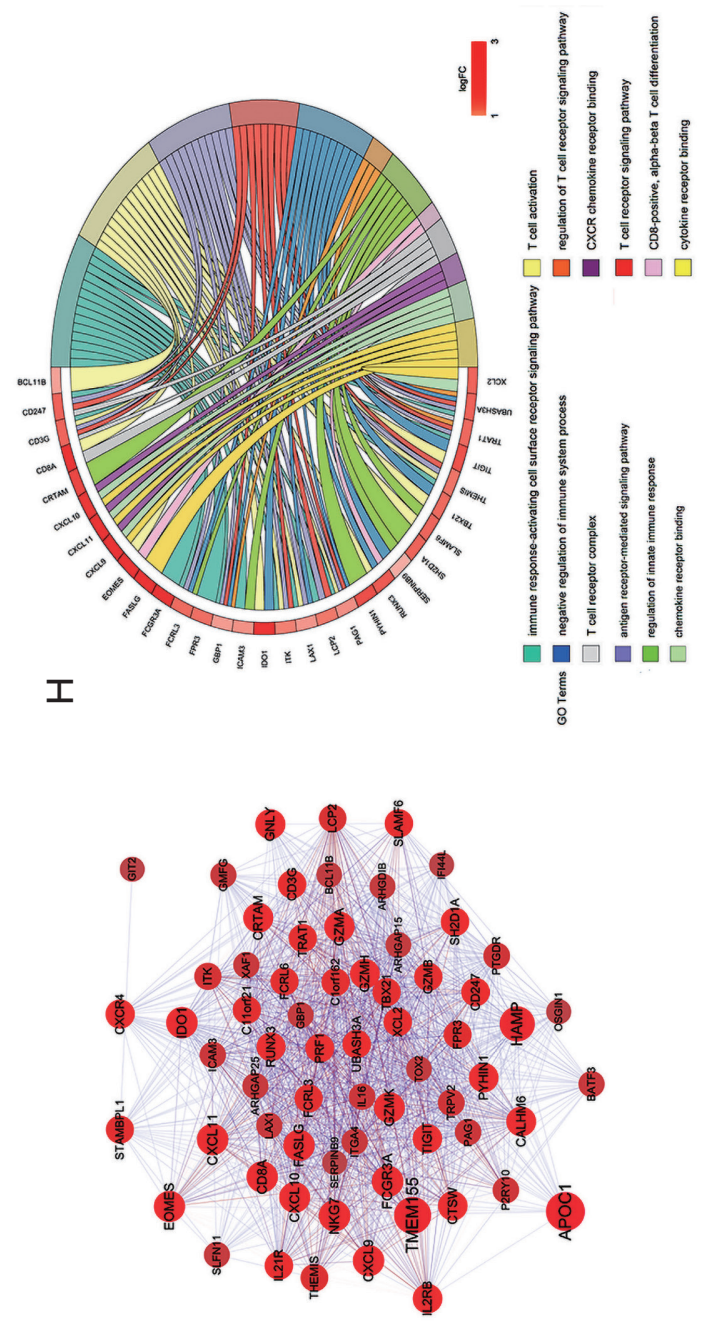

$\circlearrowleft$

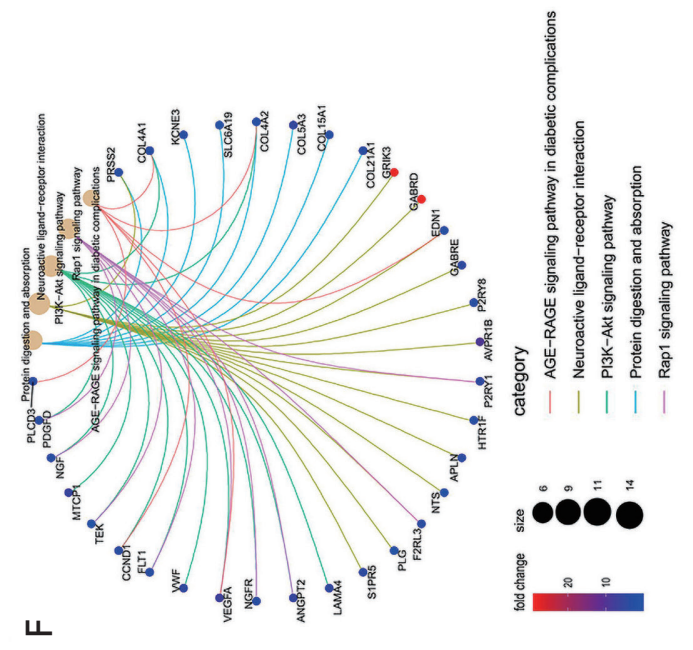

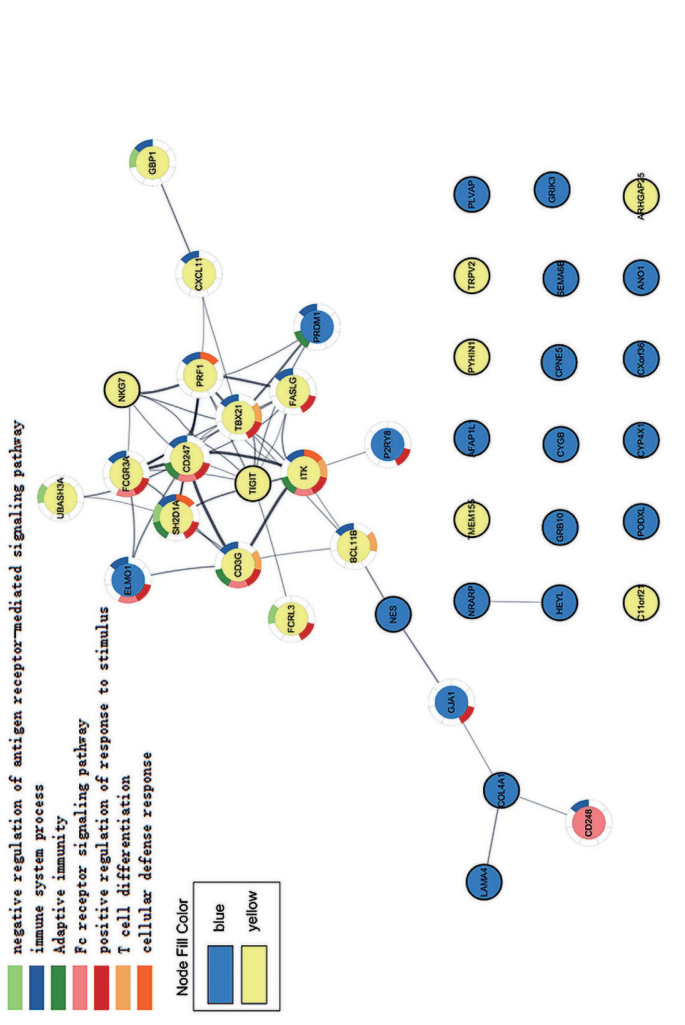

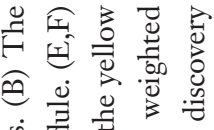

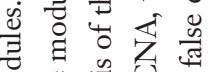

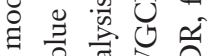

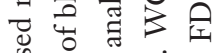

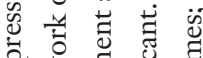

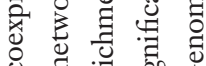

4 己

○

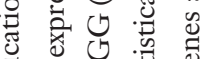

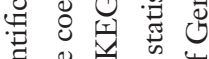

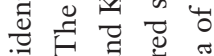

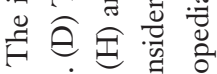

ङ

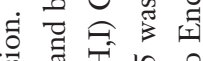

क्ष

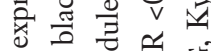

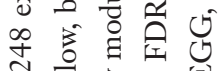

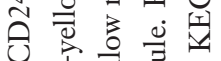

乙

닿

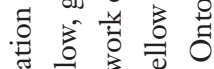

苛竞

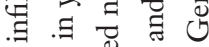

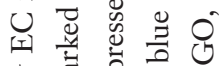

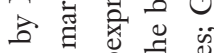

i)

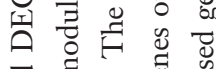

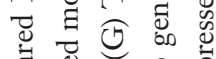

जै

Ð

पे ठै

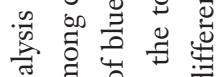

节 岕

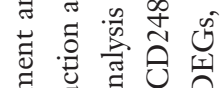

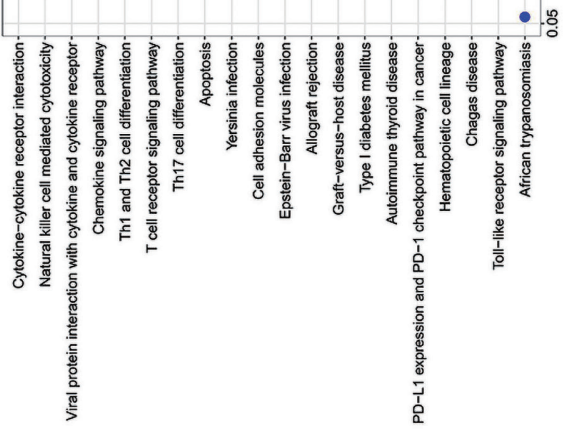

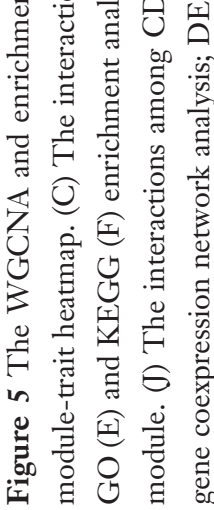



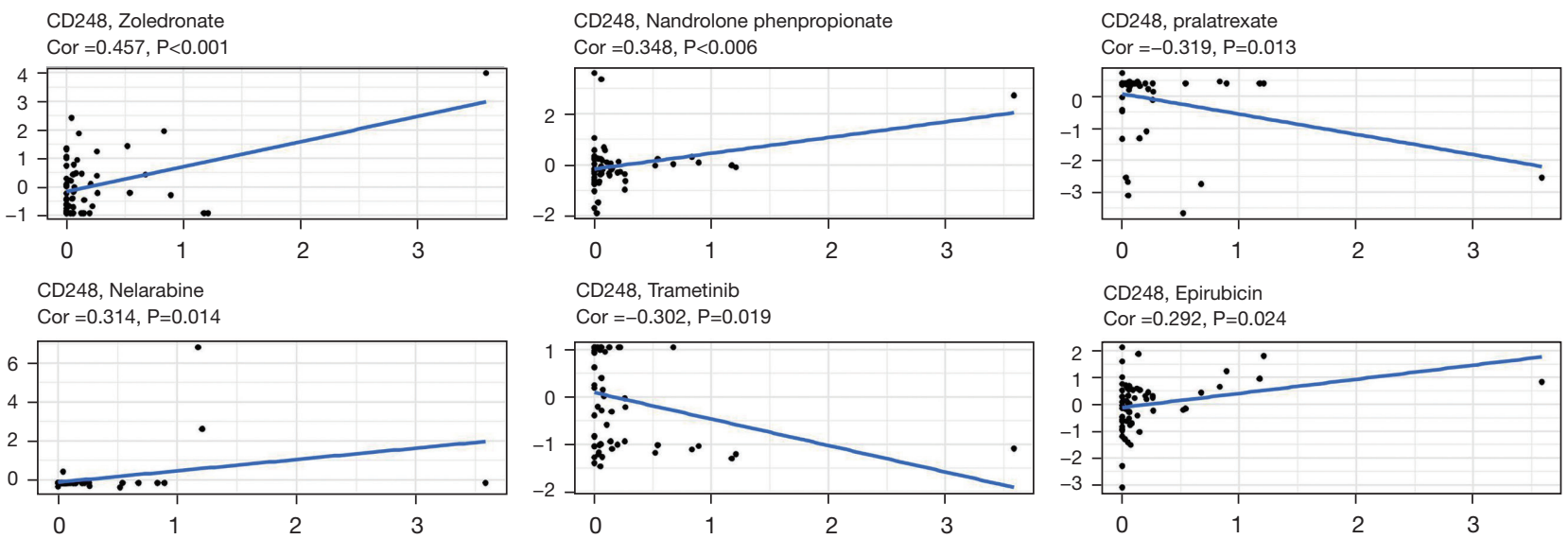

CD248, Trametinib

Cor $=-0.302, P=0.019$

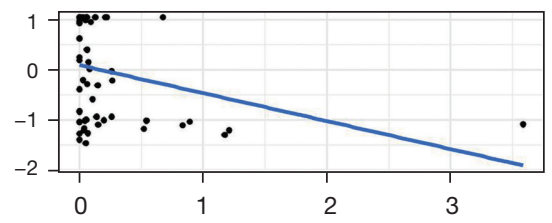

CD248, Epirubicin

Cor $=0.292, P=0.024$

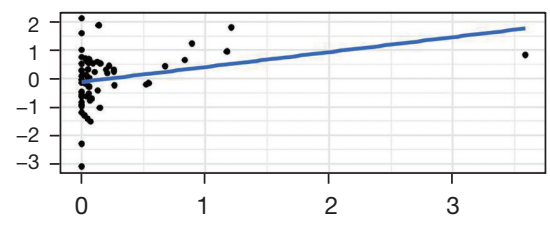

CD248, Fluphenazine

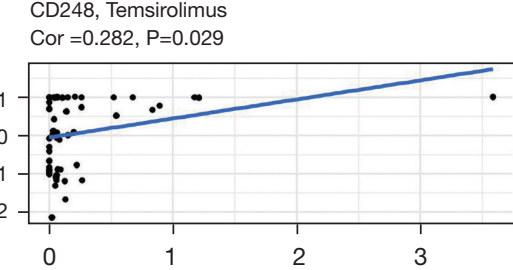

Cor $=0.278, P=0.031$

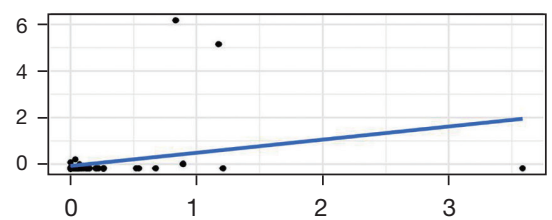

CD248, 7-ethyl-10-hydroxycamptothecin

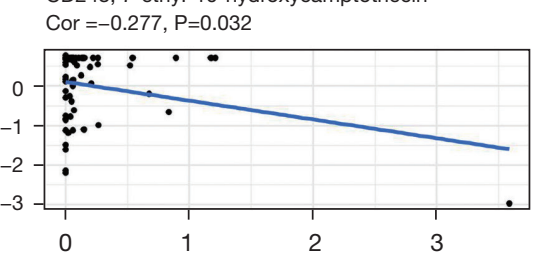

CD248, Cobimetinib (isomer 1)

Cor $=-0.274, P=0.034$

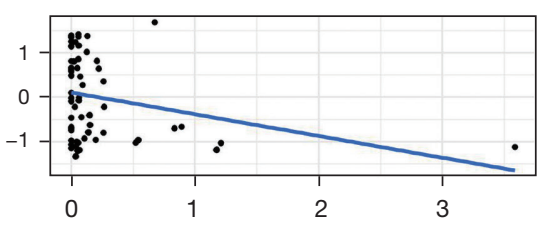

CD248, Vismodegib

Cor $=-0.257, \mathrm{P}=0.048$

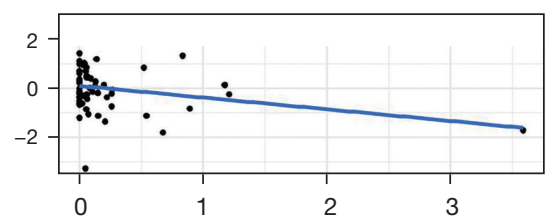

Figure 6 Prediction for clinically approved drugs responding to CD248. The correlation between CD248 and clinically approved drugs. Drugs with a $\mathrm{P}<0.05$ were considered significant. The correlation coefficient is marked in the figure.

in sarcoma and melanoma $(26,27)$. DNA vaccine targeting of CD248 has confirmed that the vaccine TEM1-TT can induce $\mathrm{CD} 8^{+}$cytotoxic $\mathrm{T}$ cell response against murine tumor-specific antigens (28). The sensitivity of CD248 in predicting OS has been published in our previous article. In short, a CD248-based prognostic signature has an excellent ability to predict the prognosis of patients with AUC $=0.889$ (16). Therefore, CD248 might bridge the gap between angiogenesis and immunosuppression, and thus be a promising prognostic and therapeutic target for RCC.

To explore the underlying mechanism of vascularCD248 in immune regulation and tumor promotion, we first demonstrated that overexpression of CD248 in tumor vasculature is associated with poor RCC survival outcome. Additionally, CD248 was found to be involved in vasculature development signaling pathways and several typical proangiogenic factors such as VEGFA, indicating that CD248 contributes to RCC progression through proangiogenic signals. Grouped by CD248 expression, we further performed IHC staining for infiltrated T cells and M2 macrophages and the results was corresponding to that grouped by ECs expression, which indicated that CD248 was an ideal target for vascularization in RCC. CD248 was also associated with other infiltrated immunosuppressive cells like monocytic lineage and MDCs, whereas relevant proof studies are lacking. WGCNA and enrichment analysis demonstrated that vascular-CD248-related DEGs could be divided into 4 prognostic gene modules, among which angiogenesis and immune-regulation-related modules were selected and visualized. Interestingly, 7 angiogenesis-related and 15 immune-regulation-related hub genes were found to interact with CD248. Therefore, further investigation for these hub genes and signaling pathways might give insight into the regulatory mechanism between angiogenesis and immune regulation.

Based on the aforementioned results, CD248 appears 
to be a promising prognostic and therapeutic target for RCC. Using the CellMiner database, we screened several clinically approved drugs that might inhibit CD248mediated tumor promotion. The candidates with statistical significance might guide RCC therapy to a certain extent, especially those drugs that respond positively to CD248, including zoledronate, nelarabine, epirubicin, and temsirolimus. Zoledronate has demonstrated positive effects on skeletal-related events in patients with RCC and bone metastasis (29). Epirubicin in combination with lexatumumab was reported to exert a synergistic cytotoxicity on human RCC cells (30). Temsirolimus has also shown proven clinical benefit in patients with nonclear cell RCC (31). Meanwhile, nelarabine, a purine analogue applied in the treatment of lymphoma or $\mathrm{T}$ cell lymphoblastic leukemia, warrants further clinical trials to explore its effects on RCC patients with high expression of CD248 (32). Additionally, CD248-based targeting therapy might be a potent antitumor strategy. Ontuxizumab (MORAB-004), a monoclonal antibody targeting CD248, has proven to be effective in metastatic melanoma in phase I and II clinical trials $(27,33)$. Yuan et al. isolated a singlechain variable fragment 78 (scFV78) against CD248 from a yeast display scFV library and showed that this might be useful for immunotoxin-based therapy in CD248-positive solid tumors (34). scFV78-based fully human antibody IgG78 has also exhibited antitumor effects in patients with hepatocellular carcinoma (15).

In conclusion, vascular-expressed CD248 contributes to RCC progression through angiogenesis and suppressive immune regulation, and may thus be a promising prognostic and therapeutic target for RCC. Targeting CD248 may prove to be a novel antitumor strategy in reversing an immunosuppressive TME. Further investigation of dualrelated hub genes and pathways might offer insight into the regulatory mechanism between angiogenesis and immunosuppression.

\section{Acknowledgments}

We acknowledge Pathology Department of Xijing Hospital, Fourth Military Medical University for the preparation of the paraffin-embedded RCC tissues and adjacent normal tissues.

Funding: This study was partly supported by National Natural Science Foundation of China (No. 81772734).

\section{Footnote}

Reporting Checklist: The authors have completed the REMARK reporting checklist. Available at https://dx.doi. org/10.21037/atm-21-6271

Data Sharing Statement: Available at https://dx.doi. org/10.21037/atm-21-6271

Conflicts of Interest: All authors have completed the ICMJE uniform disclosure form (available at https://dx.doi. org/10.21037/atm-21-6271). The authors report that this study was partly supported by National Natural Science Foundation of China (No. 81772734). The authors have no other conflicts of interest to declare.

Ethical Statement: The authors are accountable for all aspects of the work in ensuring that questions related to the accuracy of any part of the work are appropriately investigated and resolved. All procedures performed in this study involving human participants were in accordance with the Declaration of Helsinki (as revised in 2013). The study was approved by the Ethics Committee of the Xijing Hospital, Fourth Military Medical University (No. KY20162088-1). Individual consent for this retrospective analysis was waived.

Open Access Statement: This is an Open Access article distributed in accordance with the Creative Commons Attribution-NonCommercial-NoDerivs 4.0 International License (CC BY-NC-ND 4.0), which permits the noncommercial replication and distribution of the article with the strict proviso that no changes or edits are made and the original work is properly cited (including links to both the formal publication through the relevant DOI and the license). See: https://creativecommons.org/licenses/by-nc-nd/4.0/.

\section{References}

1. Roma-Rodrigues C, Mendes R, Baptista PV, et al. Targeting Tumor Microenvironment for Cancer Therapy. Int J Mol Sci 2019;20:840.

2. Sounni NE, Noel A. Targeting the tumor microenvironment for cancer therapy. Clin Chem 2013;59:85-93.

3. Anderson NM, Simon MC. The tumor microenvironment. 
Curr Biol 2020;30:R921-5.

4. Yi M, Jiao D, Qin S, et al. Synergistic effect of immune checkpoint blockade and anti-angiogenesis in cancer treatment. Mol Cancer 2019;18:60.

5. Pan H, Yu T, Sun L, et al. LncRNA FENDRR-mediated tumor suppression and tumor-immune microenvironment changes in non-small cell lung cancer. Transl Cancer Res 2020;9:3946-59.

6. Fukumura D, Kloepper J, Amoozgar Z, et al. Enhancing cancer immunotherapy using antiangiogenics: opportunities and challenges. Nat Rev Clin Oncol 2018;15:325-40.

7. Heidegger I, Pircher A, Pichler R. Targeting the Tumor Microenvironment in Renal Cell Cancer Biology and Therapy. Front Oncol 2019;9:490.

8. Motzer RJ, Escudier B, McDermott DF, et al. Nivolumab versus Everolimus in Advanced Renal-Cell Carcinoma. N Engl J Med 2015;373:1803-13.

9. Christian S, Winkler R, Helfrich I, et al. Endosialin (Tem1) is a marker of tumor-associated myofibroblasts and tumor vessel-associated mural cells. Am J Pathol 2008;172:486-94.

10. Bagley RG, Honma N, Weber W, et al. Endosialin/TEM $1 / \mathrm{CD} 248$ is a pericyte marker of embryonic and tumor neovascularization. Microvasc Res 2008;76:180-8.

11. Tomkowicz B, Rybinski K, Sebeck D, et al. Endosialin/ TEM-1/CD248 regulates pericyte proliferation through PDGF receptor signaling. Cancer Biol Ther 2010;9:908-15.

12. Simonavicius N, Ashenden M, van Weverwijk A, et al. Pericytes promote selective vessel regression to regulate vascular patterning. Blood 2012;120:1516-27.

13. Ugel S, Facciponte JG, De Sanctis F, et al. Targeting tumor vasculature: expanding the potential of DNA cancer vaccines. Cancer Immunol Immunother 2015;64:1339-48.

14. Viski C, König C, Kijewska M, et al. EndosialinExpressing Pericytes Promote Metastatic Dissemination. Cancer Res 2016;76:5313-25.

15. Yang F, Wei Y, Han D, et al. Interaction with CD68 and Regulation of GAS6 Expression by Endosialin in Fibroblasts Drives Recruitment and Polarization of Macrophages in Hepatocellular Carcinoma. Cancer Res 2020;80:3892-905.

16. Zhang K, Xu C, Liu S, et al. The Diagnostic and Immunotherapeutic Value of CD248 in Renal Cell Carcinoma. Front Oncol 2021;11:644612.

17. Bray F, Ferlay J, Soerjomataram I, et al. Global cancer statistics 2018: GLOBOCAN estimates of incidence and mortality worldwide for 36 cancers in 185 countries. CA
Cancer J Clin 2018;68:394-424.

18. Drake CG, Stein MN. The Immunobiology of Kidney Cancer. J Clin Oncol 2018. [Epub ahead of print].

19. Huang Y, Goel S, Duda DG, et al. Vascular normalization as an emerging strategy to enhance cancer immunotherapy. Cancer Res 2013;73:2943-8.

20. Remark R, Alifano M, Cremer I, et al. Characteristics and clinical impacts of the immune environments in colorectal and renal cell carcinoma lung metastases: influence of tumor origin. Clin Cancer Res 2013;19:4079-91.

21. Hinshaw DC, Shevde LA. The Tumor Microenvironment Innately Modulates Cancer Progression. Cancer Res 2019;79:4557-66.

22. Kovaleva OV, Samoilova DV, Shitova MS, et al. Tumor Associated Macrophages in Kidney Cancer. Anal Cell Pathol (Amst) 2016;2016:9307549.

23. Valdez Y, Maia M, Conway EM. CD248: reviewing its role in health and disease. Curr Drug Targets 2012;13:432-9.

24. Tomkowicz B, Rybinski K, Foley B, et al. Interaction of endosialin/TEM1 with extracellular matrix proteins mediates cell adhesion and migration. Proc Natl Acad Sci U S A 2007;104:17965-70.

25. Maia M, DeVriese A, Janssens T, et al. CD248 facilitates tumor growth via its cytoplasmic domain. BMC Cancer 2011;11:162.

26. Guo Y, Hu J, Wang Y, et al. Tumour endothelial marker 1/endosialin-mediated targeting of human sarcoma. Eur J Cancer 2018;90:111-21.

27. D'Angelo SP, Hamid OA, Tarhini A, et al. A phase 2 study of ontuxizumab, a monoclonal antibody targeting endosialin, in metastatic melanoma. Invest New Drugs 2018;36:103-13.

28. Facciponte JG, Ugel S, De Sanctis F, et al. Tumor endothelial marker 1-specific DNA vaccination targets tumor vasculature. J Clin Invest 2014;124:1497-511.

29. Tunn UW, Stenzl A, Schultze-Seemann W, et al. Positive effects of zoledronate on skeletal-related events in patients with renal cell cancer and bone metastases. Can J Urol 2012;19:6261-7.

30. Jin $\mathrm{X}, \mathrm{Wu} \mathrm{XX}$, Jin C, et al. Delineation of apoptotic genes for synergistic apoptosis of lexatumumab and anthracyclines in human renal cell carcinoma cells by polymerase chain reaction array. Anticancer Drugs 2012;23:445-54.

31. Hudes G, Carducci M, Tomczak P, et al. Temsirolimus, interferon alfa, or both for advanced renal-cell carcinoma. N Engl J Med 2007;356:2271-81.

32. Nelarabine. In: LiverTox: Clinical and Research 
Information on Drug-Induced Liver Injury. Bethesda (MD): National Institute of Diabetes and Digestive and Kidney Diseases, 2012.

33. Diaz LA Jr, Coughlin CM, Weil SC, et al. A first-inhuman phase I study of MORAb-004, a monoclonal antibody to endosialin in patients with advanced solid tumors. Clin Cancer Res 2015;21:1281-8.

Cite this article as: Liu S, Xu C, Zhang K, Han D, Yang F, Li Y, Zhao X, Ma S, Li H, Lu S, Lu T, Zhang J, Qin W, Wen W, Yang B. CD248 as a bridge between angiogenesis and immunosuppression: a promising prognostic and therapeutic target for renal cell carcinoma. Ann Transl Med 2021;9(23):1741. doi: 10.21037/atm-21-6271
34. Yuan X, Yang $M$, Chen $X$, et al. Characterization of the first fully human anti-TEM1 scFv in models of solid tumor imaging and immunotoxin-based therapy. Cancer Immunol Immunother 2017;66:367-78.

(English Language Editor: J. Gray) 

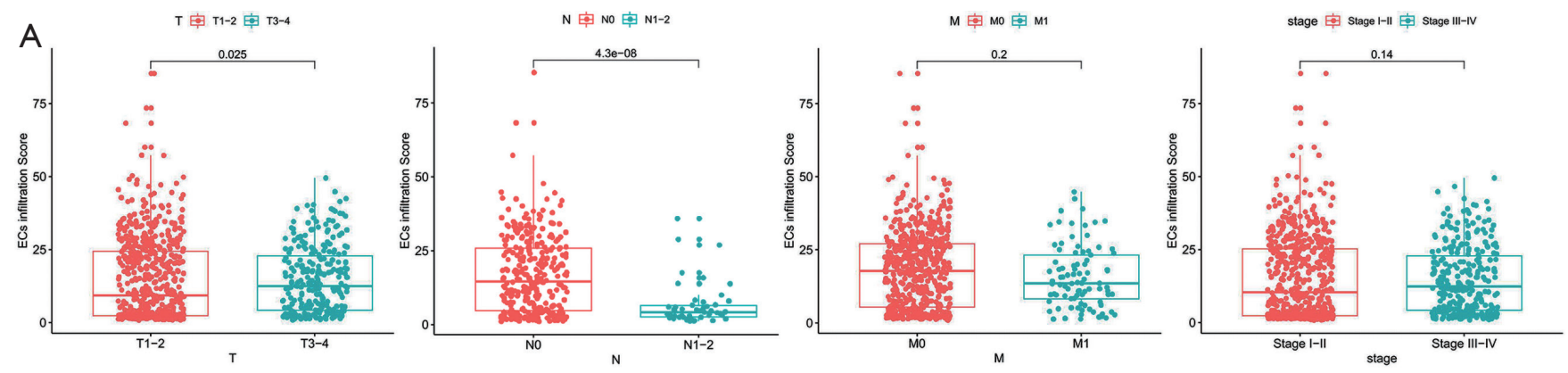

B

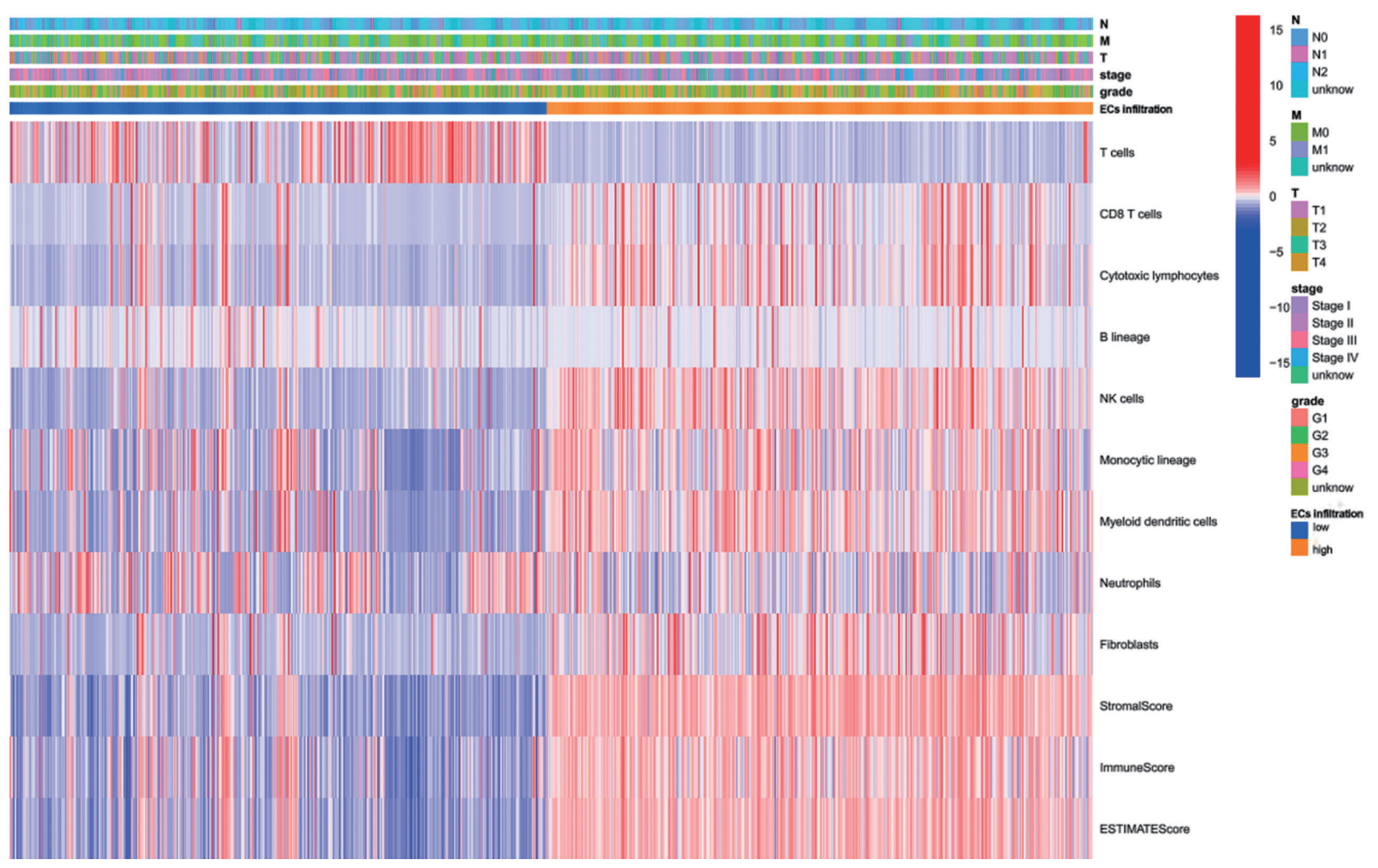

Figure S1 EC infiltration promoted RCC progression through an immunosuppressive TME. (A) Distribution of EC infiltration in TNM classification and stage of RCC. (B) Heatmap of the TME components and scores in RCC with high and low EC infiltration. EC, endothelial cells; RCC, renal cell carcinoma; TME, tumor microenvironment. 


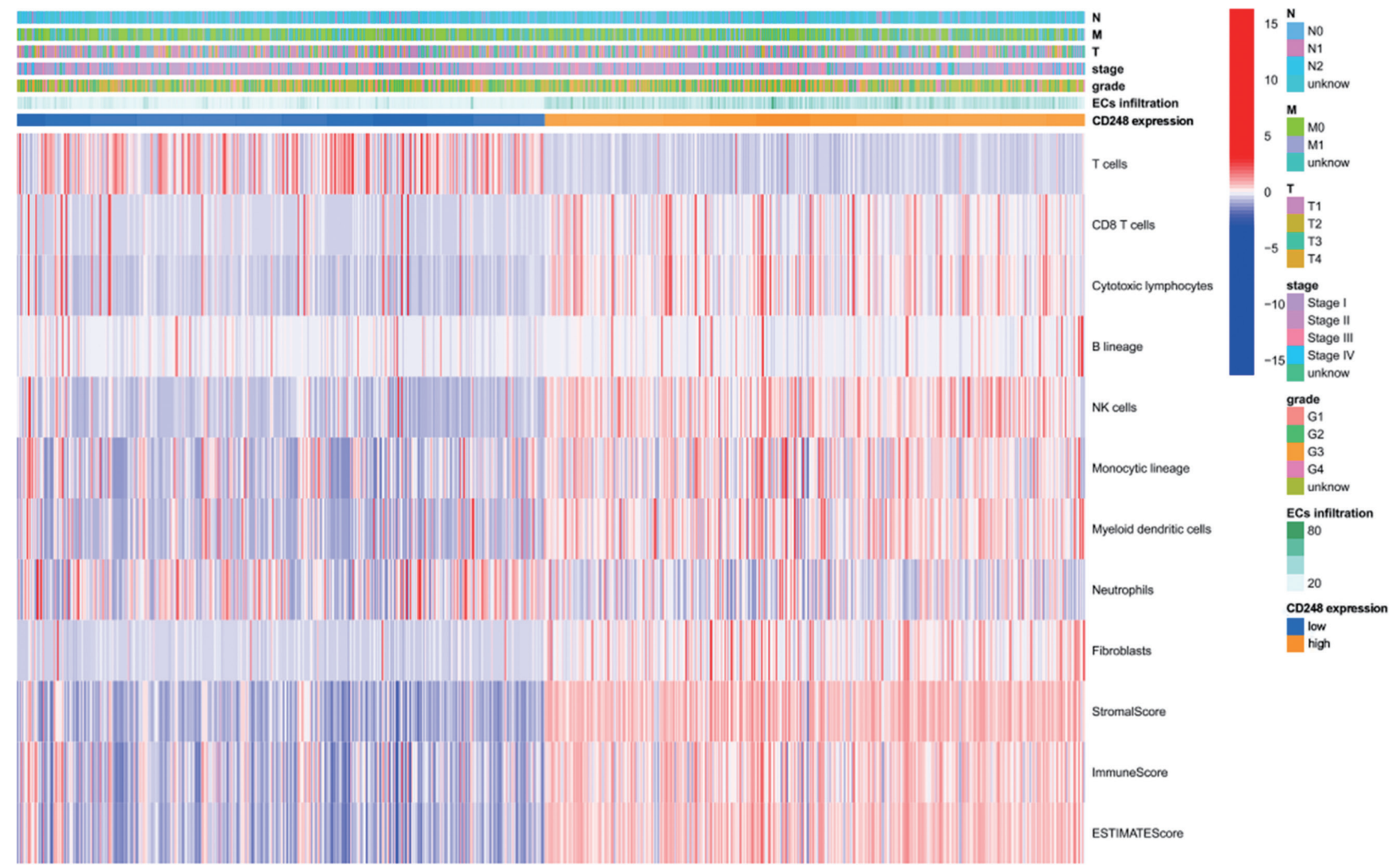

Figure S2 The heatmap of TME components and scores in RCC with high and low CD248 expression. TME, tumor microenvironment; RCC, renal cell carcinoma. 


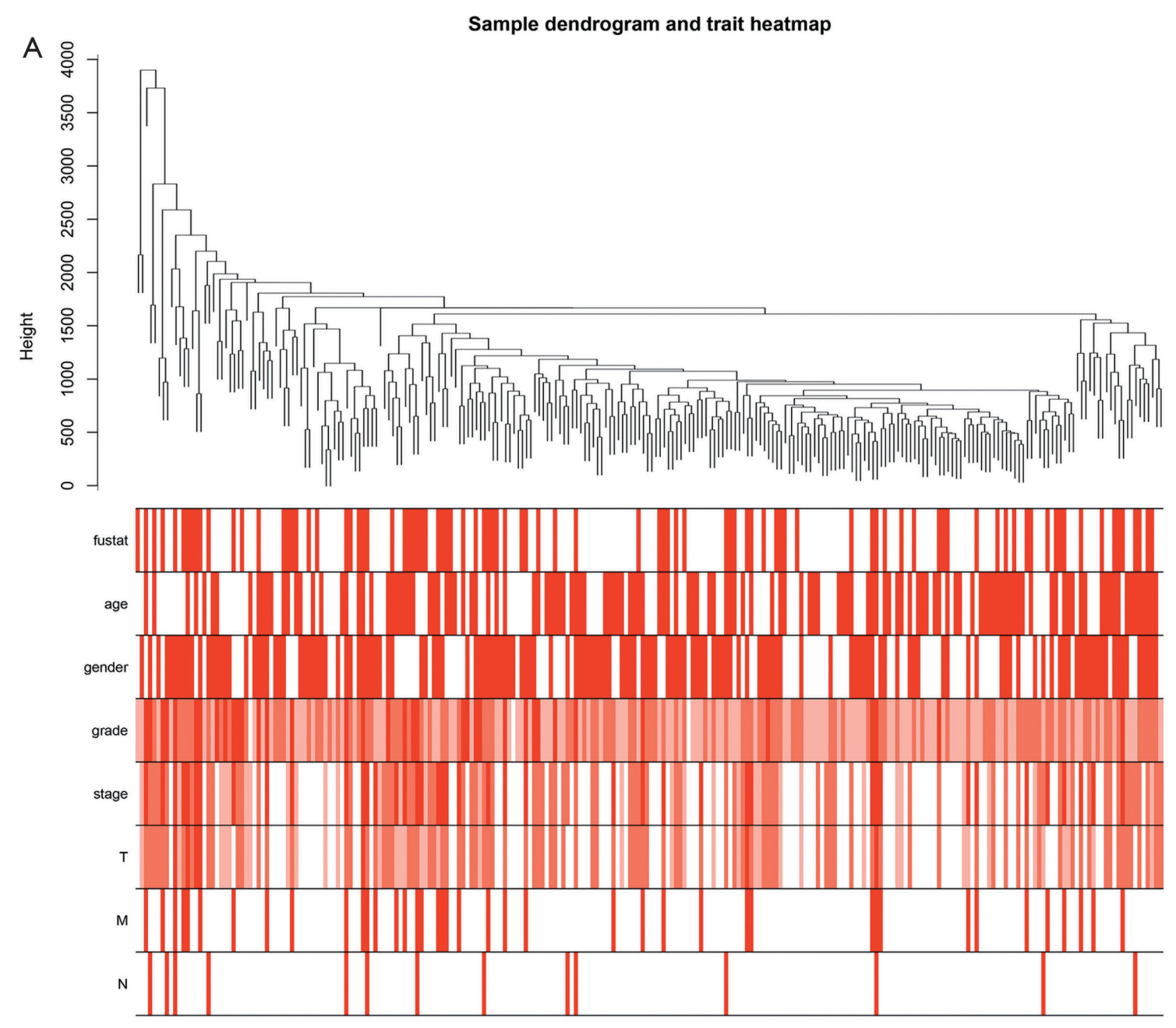

B Scale independence
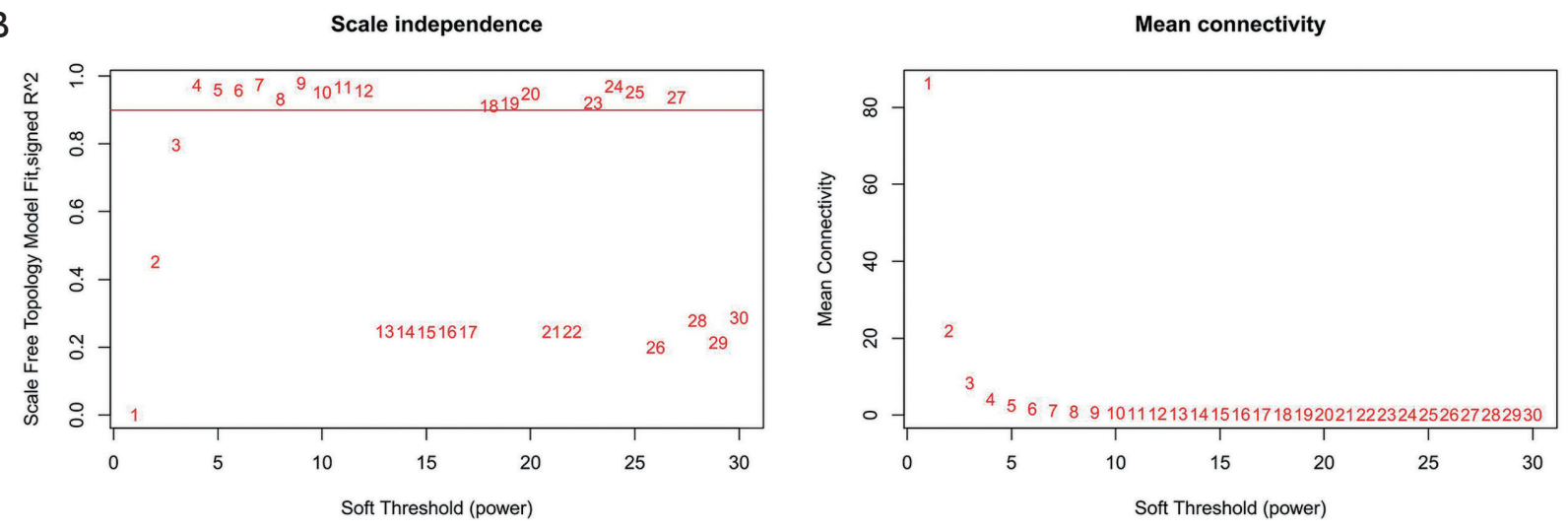

Figure S3 Analysis of the network topology for adjacency matrix weighting parameters (power). (A) Hierarchical average linkage clustering. Branches of the dendrogram represent genes with similar expression patterns. (B) The $x$-axis represents soft threshold (power), and the $y$-axis represents the scale-free fitting index and connectivity for each power. The soft-thresholding power for network construction was set at 0.9 . 

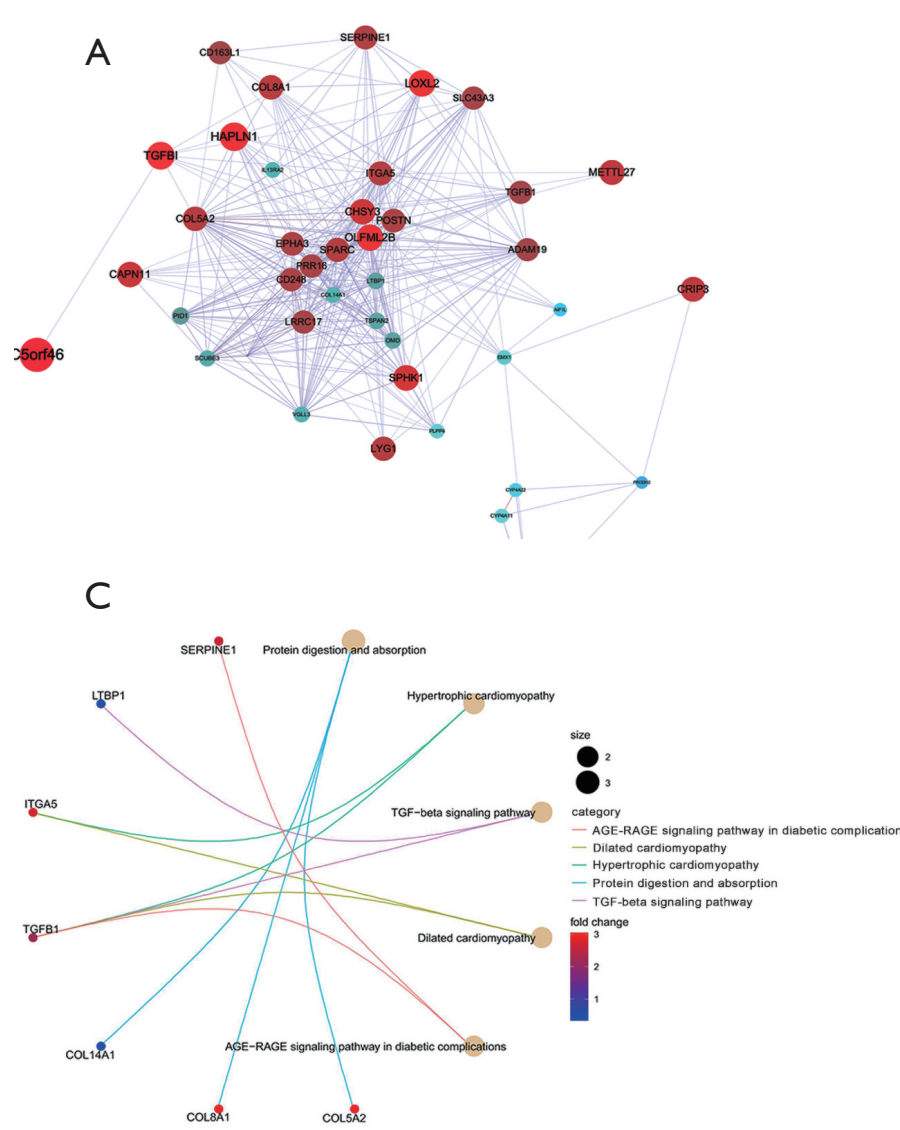

E

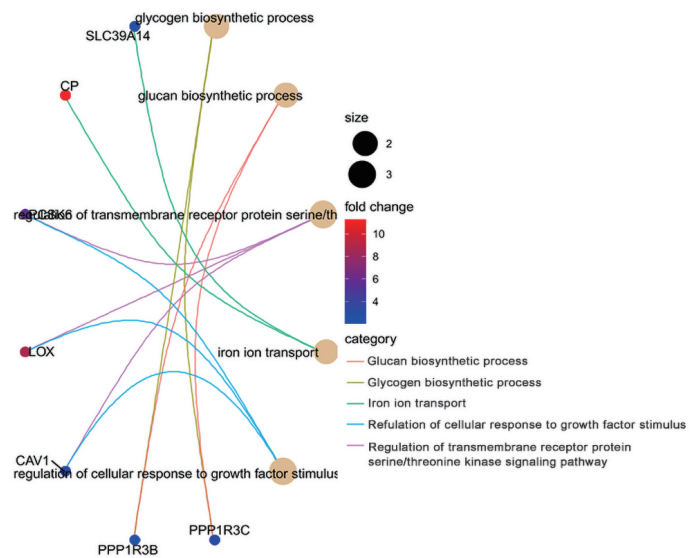

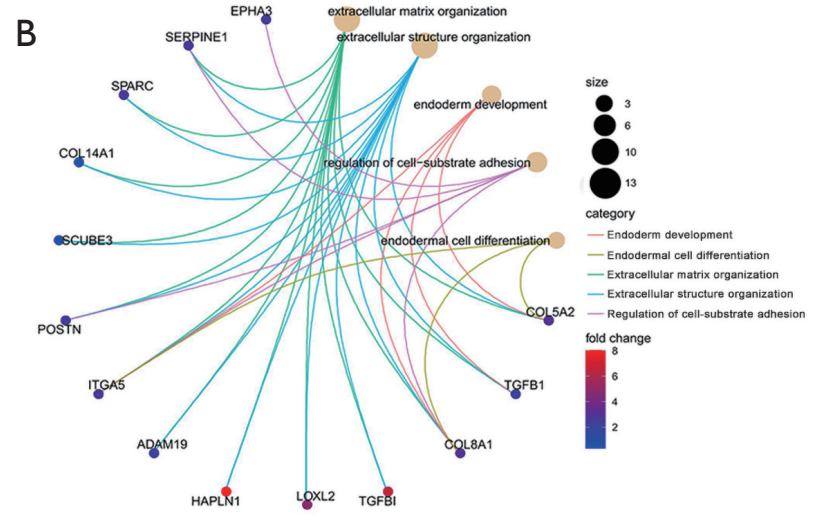

D
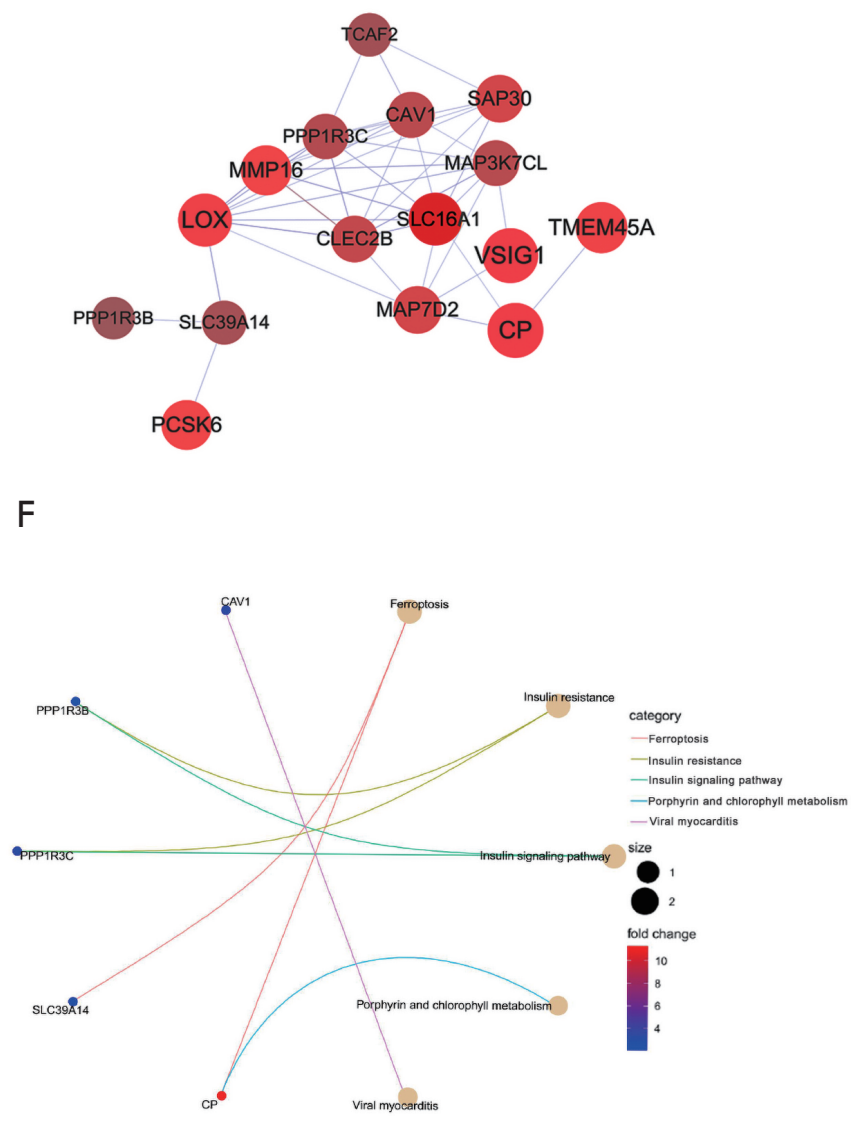

Figure S4 The WGCNA and enrichment analysis of the shared DEGs by EC infiltration and CD248 expression. (A) The coexpressed network of the black module annotated with stroma. (B,C) GO (B) and KEGG (C) enrichment analysis of the black module. (D) The coexpressed network of the green-yellow module. (E,F) GO (E) and KEGG (F) enrichment analysis of the green-yellow module. WGCNA, weighted gene coexpression network analysis; DEGs, differentially expressed genes; GO, Gene Ontology; KEGG, Kyoto Encyclopedia of Genes and Genomes. 